\title{
Boundary conditions in the Mirabelli and Peskin model
}

\author{
Dmitry V. Belyaev* \\ Department of Physics and Astronomy, The Johns Hopkins University, \\ 3400 North Charles Street, Baltimore, MD 21218, USA \\ E-mail: dmitry.belyaev@desy.de
}

\begin{abstract}
We show how the (globally supersymmetric) model of Mirabelli and Peskin can be formulated in the boundary ("downstairs" or "interval") picture. The necessary GibbonsHawking-like terms appear naturally when using (codimension one) superfields. This formulation is free of the $\delta(0)$ ambiguities of the orbifold ("upstairs") picture while describing the same physics since the boundary conditions on the fundamental domain are the same. The (natural) boundary conditions follow from the variational principle and form a closed orbit under supersymmetry variation. They reduce to the "odd $=0$ " boundary conditions in the absence of bulk-boundary coupling. We emphasize that the action is supersymmetric without the use of any boundary conditions in the off-shell formulation (but some boundary conditions are necessary for on-shell supersymmetry!).
\end{abstract}

${ }^{*}$ Present address: DESY-T, Notkestrasse 85, 22603 Hamburg, Germany 


\section{Contents}

1. Introduction 2

2. The component formulation 3

2.1 Bulk Lagrangian

2.2 Breaking $N=2$ to $N=1$ supersymmetry

2.3 Brane Lagrangian 6

3. Boundary conditions 7

3.1 Primary boundary conditions

3.2 Secondary boundary conditions 8

4. Superfield description 9

4.1 Bulk and brane Lagrangians 9

4.2 Boundary conditions in the superfield form 10

5. Boundary picture 11

5.1 The action 11

5.2 Important boundary term 12

5.3 Supersymmetry of the action 12

5.4 Variational principle 13

6. On-shell case 14

6.1 On-shell in the orbifold picture

6.2 On-shell in the boundary picture 15

6.3 On-shell closure of the supersymmetry algebra 16

6.4 The full square structure

6.5 On-shell closure of the boundary conditions 18

6.6 The role of "odd $=0$ " boundary conditions 18

7. From boundary to orbifold picture

8. Summary and Conclusions 20

A. Details of the component formulation 21

A.1 Variation of the bulk Lagrangian 21

A.2 Two-component spinor notation 22

A.3 $S U(2)$ rotation 22

A.4 $N=1$ supersymmetry and the gauge transformation 23

A.5 Orbifold equations of motion 24 
B. Superfields 25

B.1 Supersymmetry and gauge transformations 25

B.2 Component expansions 26

B.3 Boundary condition superfield 27

\section{Introduction}

In the last decade there was a revival of interest in theories on a manifold with boundary. It started from a ground-breaking paper by Horava and Witten [1], who showed that elevendimensional supergravity on a manifold with boundary appears as one of the low energy limits of the heterotic string theory. Soon after, Mirabelli and Peskin [2] introduced a simplified construct of a five-dimensional globally supersymmetric Yang-Mills theory coupled to a four-dimensional hypermultiplet living on the boundary. They argued that the analysis of this simpler system can provide useful insights into various aspects of the more complicated Horava-Witten theory.

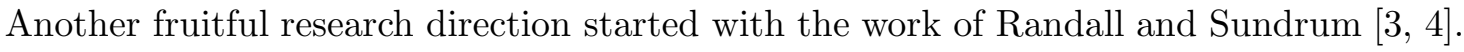
They showed that if our four-dimensional world is localized on a three-brane in a fivedimensional space-time with negative cosmological constant, then there is an attractive geometrical solution to the weak/Planck hierarchy problem. This scenario was later supersymmetrized [5, 6, 7]. In Ref. [8] it was shown how to unify the original approaches to the supersymmetric Randall-Sundrum scenario, and the issue of supersymmetric boundary conditions was addressed.

The original idea for the work presented here and in the companion paper, Ref. [9], was to understand why the use of boundary conditions was essential in the supersymmetric RandallSundrum scenario, but apparently was not important in the Mirabelli and Peskin model. The results of this research show that both statements require some adjustments. In this paper we will show that the use of some boundary conditions is required for supersymmetry of the Mirabelli and Peskin model, while in Ref. [9] we find that supersymmetry of the RandallSundrum scenario requires the use of fewer boundary conditions than was previously assumed.

Another motivation for this work was to understand the structure of boundary conditions in the Mirabelli and Peskin model, in particular, their closure under supersymmetry. We present here a set of Neumann-like boundary conditions (which we call "natural" following Ref. [10]), which guarantee that the general variation of the action vanishes for arbitrary variations of the fields on the boundary. (That is the boundary conditions are derived exactly as the bulk equations of motion.) These natural boundary conditions reduce to "odd $=0$ " ones (the vanishing of the odd fields on the boundary) only when there is no coupling of the bulk fields to the boundary. We emphasize that it is inconsistent to assume the "odd $=0$ " boundary conditions when the coupling is present (unless it is only a zeroth-order approximation in the perturbative calculations). 
As was explained by Horava and Witten [1], any theory on a manifold with boundary can be equivalently described as a theory on an orbifold, when a mirror image of the fundamental domain is introduced with the corresponding identifications. This leads to two descriptions of the same theory: the "downstairs" (boundary) picture and the "upstairs" (orbifold) picture. In the boundary picture we speak of "boundary," in the orbifold picture we have a "brane" (a set of fixed points of the mirror reflection). Both pictures have some advantages, but the boundary picture is more fundamental.

The Mirabelli and Peskin model [2] was originally set up in the orbifold picture. One of the results of Ref. [2] was to provide an algorithm for coupling bulk fields to brane-localized fields in a supersymmetric way. In this paper, we show how the model can be formulated in the boundary picture. We eliminate the mirror image space and work directly on the fundamental domain with boundary. The boundary conditions follow, as in the orbifold picture, from the variational principle provided we include a special Gibbons-Hawking-like boundary term 11] (we call it " $Y$-term" to acknowledge the work of York [12, 13]). This boundary term is also required by supersymmetry. We show how the $Y$-term follows naturally when using the codimension one $(D=4, N=1)$ superfields, which makes them very useful in constructing supersymmetric bulk-boundary couplings. Eliminating auxiliary fields, one then obtains onshell formulation which is free of $\delta(0)$ ambiguities present in the orbifold picture.

The paper is organized as follows. In Section 2, we review the original formulation of the Mirabelli and Peskin model: off-shell, in components, on the orbifold. In Section 3, we derive the natural boundary conditions and discuss their closure under supersymmetry. In Section 1 , we turn to the superfield formulation of the model and show how the boundary conditions are reproduced. In Section 5, we present the formulation of the model in the boundary picture. We discuss there the derivation and the role of the $Y$-term. In Section 6 , we discuss the on-shell case (when the auxiliary fields of the model are eliminated). There we show that in the orbifold picture, as in the off-shell case, we still do not need to use any boundary conditions to establish supersymmetry, whereas in the boundary picture, the use of some boundary conditions is required. We also show that the "odd $=0$ " boundary conditions are consistent only as free field boundary conditions. Finally, in Section 0, we discuss the transition between the boundary and the orbifold pictures.

The Appendices contain some technical details, which are separated for clarity of discussion. The basic conventions are the same as in Ref. [8].

\section{The component formulation}

In this section we write the model of Mirabelli and Peskin, adjusting it to our conventions. We present the model in its original formulation: on the orbifold, in components, with off-shell field content. In the later sections we present the model in other settings: on a space-time domain with boundary, in the superfield formulation, and truncated to on-shell field content. For simplicity, we consider only the Abelian case, since it is sufficient for our purposes. 


\subsection{Bulk Lagrangian}

The bulk Lagrangian is the standard globally supersymmetric Lagrangian for an Abelian gauge multiplet, $\left(A_{M}, \Phi, \Lambda_{i}, X_{a}\right)$, in five dimensions $(M, N=\{0,1,2,3,5\}, i, j=\{1,2\}$, $a=\{1,2,3\})$,

$$
\mathcal{L}_{5}=-\frac{1}{4} F_{M N} F^{M N}-\frac{1}{2} \partial_{M} \Phi \partial^{M} \Phi-\frac{i}{2} \widetilde{\Lambda}^{i} \Gamma^{M} \partial_{M} \Lambda_{i}+\frac{1}{2} X_{a} X_{a} .
$$

Here $F_{M N}$ is the field strength for a gauge boson $A_{M}, \Phi$ is a scalar, $\Lambda_{i}$ is a symplecticMajorana spinor, and $X_{a}$ is a triplet of auxiliary fields. The Lagrangian has four global symmetries (supersymmetry, translation, Lorentz and $S U(2)$ ), and one local invariance $(U(1)$ gauge invariance). The Lorentz transformation is standard and it is not important for our discussion. The other transformations are

- (global) supersymmetry (with fermionic parameter $\mathcal{H}_{i}=$ const),

$$
\begin{aligned}
\delta_{\mathcal{H}} A^{M} & =i \widetilde{\mathcal{H}}^{i} \Gamma^{M} \Lambda_{i} \\
\delta_{\mathcal{H}} \Phi & =i \widetilde{\mathcal{H}}^{i} \Lambda_{i} \\
\delta_{\mathcal{H}} X_{a} & =\widetilde{\mathcal{H}}^{i}\left(\sigma_{a}\right)_{i}{ }^{j} \Gamma^{M} \partial_{M} \Lambda_{j} \\
\delta_{\mathcal{H}} \Lambda_{i} & =\left(\Sigma^{M N} F_{M N}+\Gamma^{M} \partial_{M} \Phi\right) \mathcal{H}_{i}+i X_{a}\left(\sigma_{a}\right)_{i}{ }^{j} \mathcal{H}_{j} ;
\end{aligned}
$$

- (global) translation (with parameter $v^{M}=$ const),

$$
\delta_{v}\left(A_{M}, \Phi, X_{a}, \Lambda_{i}\right)=v^{K} \partial_{K}\left(A_{M}, \Phi, X_{a}, \Lambda_{i}\right)
$$

- (global) $S U(2)$ rotation (with constant matrix parameter $U \in S U(2)$ ),

$$
\Lambda_{i}^{\prime}=U_{i}^{j} \Lambda_{j}, \quad X_{a}^{\prime} \sigma_{a}=U\left(X_{a} \sigma_{a}\right) U^{\dagger}
$$

- (local) $U(1)$ gauge transformation (with parameter $u(x)$ ),

$$
\delta_{u} A_{M}=\partial_{M} u .
$$

(In the above, the $\sigma_{a}=\left\{\sigma_{1}, \sigma_{2}, \sigma_{3}\right\}$ are the usual Pauli matrices.) The Lagrangian $\mathcal{L}_{5}$ is invariant $\left(\delta \mathcal{L}_{5}=0\right)$ under the $U(1), S U(2)$ and Lorentz transformations, but it changes into a total derivative under the translation and supersymmetry transformations,

$$
\delta_{v} \mathcal{L}_{5}=\partial_{M}\left(v^{M} \mathcal{L}_{5}\right), \quad \delta_{\mathcal{H}} \mathcal{L}_{5}=\partial_{M} \widetilde{K}^{M},
$$

where $\widetilde{K}^{M}$ is given in Eq. (A.4). The supersymmetry algebra has the following form,

$$
\left[\delta_{\Xi}, \delta_{\mathcal{H}}\right]=v^{K} \partial_{K}+\delta_{u}
$$

where the parameters of the translation and the $U(1)$ transformation are

$$
v^{K}=2 i\left(\widetilde{\mathcal{H}}^{i} \Gamma^{K} \Xi_{i}\right), \quad u=-2 i\left(\widetilde{\mathcal{H}}^{i} \Gamma^{K} \Xi_{i}\right) A_{K}-2 i\left(\widetilde{\mathcal{H}}^{i} \Xi_{i}\right) \Phi
$$




\subsection{Breaking $N=2$ to $N=1$ supersymmetry}

We will use the two-component spinor notation, in which the symplectic-Majorana spinors $\Lambda_{i}$ and $\mathcal{H}_{j}$ are represented by pairs of two-component spinors: $\left(\lambda_{1}, \lambda_{2}\right)$ and $\left(\eta_{1}, \eta_{2}\right)$ (see Appendix A.2). After the $M=\{m, 5\}$ split and in the two-component spinor notation, the Lagrangian assumes the following form,

$$
\begin{aligned}
\mathcal{L}_{5}= & -\frac{1}{4} F_{m n} F^{m n}-\frac{1}{2} F_{m 5} F^{m 5}-\frac{1}{2} \partial_{m} \Phi \partial^{m} \Phi-\frac{1}{2} \partial_{5} \Phi \partial_{5} \Phi+\frac{1}{2} X_{12} X_{12}^{*}+\frac{1}{2} X_{3}^{2} \\
& -\left[\frac{i}{2} \lambda_{1} \sigma^{m} \partial_{m} \bar{\lambda}_{1}+\frac{i}{2} \lambda_{2} \sigma^{m} \partial_{m} \bar{\lambda}_{2}+\frac{1}{2}\left(\lambda_{2} \partial_{5} \lambda_{1}-\lambda_{1} \partial_{5} \lambda_{2}\right)+\text { h.c. }\right]
\end{aligned}
$$

where $X_{12}=X_{1}+i X_{2}$. The Lagrangian is invariant (up to total $\partial_{m}$ and $\partial_{5}$ derivatives) under the supersymmetry transformations with arbitrary (constant) $\eta_{1}$ and $\eta_{2}$. The Lagrangian is, therefore, $N=2$ supersymmetric.

In the presence of a brane/boundary, we can keep only half of the bulk supersymmetry intact. This statement is based on the following standard argument. The commutator of two supersymmetry transformations generates a translation with parameter

$$
v^{M}=2 i\left(\widetilde{\mathcal{H}}^{i} \Gamma^{K} \Xi_{i}\right)
$$

The brane breaks translational invariance, allowing only $v^{5}=0$. (We assume that the brane/boundary is located at $x^{5}=$ const.) For the supersymmetry parameters this implies

$$
v^{5}=2\left(\eta_{2} \xi_{1}-\eta_{1} \xi_{2}\right)+\text { h.c. }=0 \text {. }
$$

Therefore, the two supersymmetry parameters have to be related,

$$
\eta_{2}=\alpha \eta_{1}
$$

where $\alpha$ is an arbitrary complex constant. This eliminates one linear combination of $\eta_{1}$ and $\eta_{2}$; the orthogonal linear combination describes the unbroken $N=1$ supersymmetry.

Note that such supersymmetry transformations still generate a non-zero $U(1)$ transformation with

$$
u=-2\left[i\left(\eta_{1} \sigma^{m} \bar{\xi}_{1}+\eta_{2} \sigma^{m} \bar{\xi}_{2}\right)+\text { h.c. }\right] A_{m}
$$

This implies that the brane/boundary action we will introduce must be gauge invariant (for the total action to be supersymmetric).

We choose to preserve $\eta_{1}$ supersymmetry, so that from now on we set

$$
\eta_{2}=0
$$

Any other choice of $\alpha$ (preserving another linear combination of $\eta_{1}$ and $\eta_{2}$ ) can be obtained by a global $S U(2)$ rotation (see Appendix A.3). 
Under the $N=1\left(\eta=\eta_{1}\right)$ supersymmetry, the five-dimensional gauge supermultiplet splits into two four-dimensional supermultiplets (see Appendix A.4), the gauge and chiral multiplets,

$$
\begin{aligned}
\left(v_{m}, \lambda, D\right) & =\left(A_{m}, \lambda_{1}, X_{3}-\partial_{5} \Phi\right) \\
\left(\phi_{2}, \psi_{2}, F_{2}\right) & =\left(\Phi+i A_{5},-i \sqrt{2} \lambda_{2},-X_{12}\right) .
\end{aligned}
$$

The bulk Lagrangian can also be written in terms of these fields,

$$
\begin{aligned}
\mathcal{L}_{5}= & -\frac{1}{4} v_{m n} v^{m n}-\frac{1}{2}\left(\partial_{5} v_{m}\right)\left(\partial_{5} v^{m}\right)-\frac{1}{2} \partial_{m} \phi_{2} \partial^{m} \phi_{2}^{*}+\frac{1}{2} F_{2} F_{2}^{*}+\frac{1}{2} D^{2} \\
& -\frac{i}{2}\left(\partial_{m} \phi_{2}-\partial_{m} \phi_{2}^{*}\right) \partial_{5} v^{m}+\frac{1}{2} D \partial_{5}\left(\phi_{2}+\phi_{2}^{*}\right) \\
& -\left(\frac{i}{2} \lambda \sigma^{m} \partial_{m} \bar{\lambda}+\frac{i}{4} \psi_{2} \sigma^{m} \partial_{m} \bar{\psi}_{2}+\frac{i}{2 \sqrt{2}}\left(\psi_{2} \partial_{5} \lambda-\lambda \partial_{5} \psi_{2}\right)+\text { h.c. }\right) .
\end{aligned}
$$

\subsection{Brane Lagrangian}

In the orbifold picture, the bulk action is invariant under supersymmetry because the total derivative terms integrate to zero. Therefore, to have a supersymmetric bulk-plus-brane system we simply need to add a supersymmetric brane action, containing couplings between the induced bulk fields and intrinsic brane fields.

We consider a four-dimensional chiral supermultiplet $(\phi, \psi, F)$ living on the brane and couple it to the bulk vector supermultiplet $\left(v_{m}, \lambda, D\right)$. The brane Lagrangian is therefore the standard four-dimensional Lagrangian [24], coupling the two multiplets in a gauge invariant way, ${ }^{1}$

$$
\mathcal{L}_{4}=-\mathcal{D}_{m} \phi \mathcal{D}^{m} \phi^{*}-i \bar{\psi} \bar{\sigma}^{m} \mathcal{D}_{m} \psi+F F^{*}+\frac{i}{\sqrt{2}}\left(\phi^{*} \lambda \psi-\phi \overline{\lambda \psi}\right)+\frac{1}{2} \phi \phi^{*} D
$$

where $\mathcal{D}_{m}=\partial_{m}+\frac{i}{2} v_{m}$.

The brane Lagrangian is invariant (up to a total $\partial_{m}$ derivative) under the standard supersymmetry transformations, see Eqs. (A.20) and (A.24). It is also invariant under the $U(1)$ gauge transformation (see Appendix A.4),

$$
\delta_{u} v_{m}=\partial_{m} u, \quad \delta_{u}(\phi, \psi, F)=-\frac{i}{2} u(\phi, \psi, F)
$$

\footnotetext{
${ }^{1}$ The coupling constant $Q$ can be made explicit by multiplying every $v_{m}, \lambda$ and $D$ in $\mathcal{L}_{4}$ by $Q$. The righthand sides of the boundary conditions, Eqs. (3.5) and (3.7), are then also multiplied by $Q$, so that turning the coupling off (setting $Q=0$ ) gives the "odd =0" boundary conditions. In the non-abelian case [2], the strength of the coupling is dependent on the coupling constant $g$ of self-interaction of the gauge fields; the decoupling happens when the brane chiral multiplets are in the trivial representation $R$ of the gauge group: $t_{a b}^{A}=0$.
} 


\section{Boundary conditions}

We will consider first the orbifold picture, where the bulk action is $\mathcal{L}_{5}$ integrated over $\mathbb{R}^{1,4}$ and the brane action is $\mathcal{L}_{4}$ integrated over the hypersurface $x^{5}=0$. In this picture, both total $\partial_{m}$ and $\partial_{5}$ derivatives can be neglected. The bulk and brane actions, therefore, are separately supersymmetric, which is true without reference to any jump/boundary conditions. However, the presence of the brane-localized sources (due to the brane action) requires certain jump conditions across the brane to be satisfied. And when we impose the $\mathbb{Z}_{2}$ symmetry, these jump conditions turn into boundary conditions on each side of the brane. In this section we derive these boundary conditions and discuss their closure under the $N=1$ supersymmetry transformations. ${ }^{2}$

\subsection{Primary boundary conditions}

In the orbifold picture, the bulk and brane Lagrangians can be combined into a total bulkplus-brane Lagrangian,

$$
\mathcal{L}=\mathcal{L}_{5}+\delta(z) \mathcal{L}_{4}
$$

where the delta-function $\delta(z)$ is localized at $z \equiv x^{5}=0$. The equations of motion for this Lagrangian are straightforward to derive and are summarized in Eq. (A.30). The $\delta(z)$-terms in the equations enforce the following jump conditions,

$$
\begin{aligned}
{\left[F_{m 5}\right] } & =-\frac{i}{2}\left(\phi \mathcal{D}_{m} \phi^{*}-\phi^{*} \mathcal{D}_{m} \phi\right)-\frac{1}{2} \psi \sigma_{m} \bar{\psi} \\
{[\Phi] } & =-\frac{1}{2} \phi \phi^{*} \\
{\left[\lambda_{2}\right] } & =-\frac{i}{\sqrt{2}} \phi^{*} \psi,
\end{aligned}
$$

where the square brackets denote the jump across the brane,

$$
[\Phi(x)] \equiv \Phi(x, z=+0)-\Phi(x, z=-0) .
$$

Let us now introduce a $\mathbb{Z}_{2}$ parity, $f(-z)=P[f] f(+z)$, according to

$$
P\left[A_{m}, \lambda_{1}, X_{3}\right]=+1, \quad P\left[A_{5}, \lambda_{2}, X_{12}, \Phi\right]=-1 .
$$

(As usual, we call $P[f]=+1$ fields "even," and $P[f]=-1$ fields "odd".) These parity assignments are consistent with the equations of motion and the supersymmetry transformations. They allow us to rewrite the jump conditions as boundary conditions for fields at $z=+0$,

$$
\begin{aligned}
& B_{1}\left(A_{m}\right): 2 F_{m 5} \stackrel{+0}{=}-\frac{i}{2}\left(\phi \mathcal{D}_{m} \phi^{*}-\phi^{*} \mathcal{D}_{m} \phi\right)-\frac{1}{2} \psi \sigma_{m} \bar{\psi} \\
& B_{1}(\Phi) \quad: 2 \Phi \quad \stackrel{+0}{=}-\frac{1}{2} \phi \phi^{*} \\
& B_{1}\left(\lambda_{1}\right): 2 \lambda_{2} \stackrel{+0}{=}-\frac{i}{\sqrt{2}} \phi^{*} \psi .
\end{aligned}
$$

\footnotetext{
${ }^{2}$ The closure under supersymmetry of boundary conditions in various supersymmetric theories was discussed before. Some of the early references are Refs. [14, 15]. For a more recent discussion see Refs. 16, 17].
} 
The bulk-plus-brane equations of motion split into bulk equations of motion (for the bulk fields), brane equations of motion (for the brane fields, both intrinsic and induced from the bulk) and boundary conditions (relating near-brane values of the bulk fields to the brane fields). For a general variation of the bulk-plus-brane action to vanish, all the equations of motion and the boundary conditions must be satisfied. On the other hand, the variation of the bulk-plus-brane action under the supersymmetry transformations vanishes without the use of either equations of motion or boundary conditions.

\subsection{Secondary boundary conditions}

If supersymmetry is a true symmetry of the bulk-plus-brane system, the boundary conditions should also be invariant under the supersymmetry transformations. The primary boundary conditions $B_{1}\left(A_{m}\right), B_{1}(\Phi)$ and $B_{1}\left(\lambda_{1}\right)$ do not form a supersymmetric system of equations. However, after a finite number of the supersymmetry variations, we arrive at a supersymmetric system including both the primary and secondary boundary conditions.

The structure of the supersymmetry variation for this system of boundary conditions is as follows,

$$
\begin{aligned}
& \delta_{\eta} B_{1}(\Phi)=B_{1}\left(\lambda_{1}\right) \\
& \delta_{\eta} B_{1}\left(\lambda_{1}\right)=B_{1}\left(A_{m}\right) \oplus B_{2}\left(\lambda_{1}\right) \oplus B_{1}(\Phi) \\
& \delta_{\eta} B_{2}\left(\lambda_{1}\right)=B_{2}\left(A_{m}\right) \oplus B_{1}\left(\lambda_{1}\right) \\
& \delta_{\eta} B_{1}\left(A_{m}\right)=B_{2}\left(A_{m}\right) \oplus B_{1}\left(\lambda_{1}\right) \\
& \delta_{\eta} B_{2}\left(A_{m}\right)=B_{3}\left(A_{m}\right) \oplus B_{1}\left(A_{m}\right) \\
& \delta_{\eta} B_{3}\left(A_{m}\right)=B_{2}\left(A_{m}\right)
\end{aligned}
$$

The secondary boundary conditions are

$$
\begin{aligned}
& B_{2}\left(A_{m}\right): 2 \partial_{5} \lambda_{1} \stackrel{+0}{=} \frac{1}{\sqrt{2}} \sigma^{m} \bar{\psi} \mathcal{D}_{m} \phi+\frac{i}{\sqrt{2}} \psi F^{*}+\frac{1}{2} \phi \phi^{*} \lambda_{1} \\
& B_{2}\left(\lambda_{1}\right): 2 X_{12} \stackrel{+0}{=} F \phi^{*} \\
& B_{3}\left(A_{m}\right): 2 \partial_{5} D \stackrel{+0}{=} \mathcal{L}_{4}^{r},
\end{aligned}
$$

where $\mathcal{L}_{4}^{r}$ is real and differs from $\mathcal{L}_{4}$ by a total $\partial_{m}$ derivative,

$$
\begin{aligned}
\mathcal{L}_{4}^{r}= & -\mathcal{D}_{m} \phi \mathcal{D}^{m} \phi^{*}+F F^{*}+\frac{1}{2} \phi \phi^{*} D \\
& -\frac{i}{2}\left(\psi \sigma^{m} \mathcal{D}_{m} \bar{\psi}-\mathcal{D}_{m} \psi \sigma^{m} \bar{\psi}\right)+\frac{i}{\sqrt{2}}\left(\phi^{*} \lambda \psi-\phi \overline{\lambda \psi}\right) .
\end{aligned}
$$

Note that the boundary conditions are gauge invariant.

The fact the the boundary conditions are closed under supersymmetry implies that they can be cast in a superfield form. This is indeed so, as will be shown in the next section. 


\section{Superfield description}

$N=1$ supersymmetry can be conveniently described in terms of superfields [24]. For the Mirabelli and Peskin model, the superfield description of the unbroken $N=1$ supersymmetry was discussed in Ref. [18]. In this section, we use this description to cast our boundary conditions in a superfield form. We also find that the superfield description leads to a bulk Lagrangian $\mathcal{L}_{5}{ }^{\prime}$ different from $\mathcal{L}_{5}$ by a total derivative term, which produces the necessary boundary Lagrangian in the boundary picture.

\subsection{Bulk and brane Lagrangians}

The $N=2$ five-dimensional supersymmetric theory can be described in terms of $N=1$ four-dimensional superfields. The vector supermultiplet $\left(v_{m}, \lambda, D\right)$ and chiral supermultiplets $(\phi, \psi, F)$ and $\left(\phi_{2}, \psi_{2}, F_{2}\right)$ are described by a real vector superfield $\mathbf{V}$ (in the WZ gauge) and chiral superfields $\boldsymbol{\Phi}$ and $\boldsymbol{\Phi}_{\mathbf{2}}$, respectively. The component expansions are given in Appendix B.1. The gauge transformation, Eqs. A.23) and (A.26), generalizes to a supergauge transformation, parametrized by a chiral superfield $\boldsymbol{\Lambda}$,

$$
\delta \mathbf{V}=\boldsymbol{\Lambda}+\boldsymbol{\Lambda}^{\dagger}, \quad \delta \Phi=-\Lambda \Phi, \quad \delta \Phi_{2}=\mathbf{2} \partial_{5} \Lambda
$$

Let us consider a supergauge invariant Lagrangian that can be built from the superfields $\mathbf{V}$ and $\boldsymbol{\Phi}_{\mathbf{2}}$,

$$
\mathcal{L}_{5}{ }^{\prime}=\frac{1}{4} \int d^{2} \theta \mathbf{W} \mathbf{W}+\text { h.c. }+\int d^{2} \theta d^{2} \bar{\theta} \mathbf{Z}^{2}
$$

where $\mathbf{W}$ is the field strength for $\mathbf{V}$ (see Ref. [24]), and

$$
\mathbf{Z}=\partial_{5} \mathbf{V}-\frac{1}{2}\left(\boldsymbol{\Phi}_{\mathbf{2}}+\boldsymbol{\Phi}_{\mathbf{2}}^{\dagger}\right)
$$

is defined following Ref. [19]. Both $\mathbf{W}$ and $\mathbf{Z}$ are invariant under the supergauge transformation, Eq. (4.1). Expanding in components and comparing with Eq. (2.17), we find

$$
\mathcal{L}_{5}{ }^{\prime}=\mathcal{L}_{5}+\partial_{5}\left(-\frac{1}{2}\left(\phi_{2}+\phi_{2}^{*}\right) D-\frac{i}{2 \sqrt{2}}\left(\lambda \psi_{2}-\overline{\lambda \psi}_{2}\right)\right)+\frac{1}{16} \partial_{m} \partial^{m}\left(\phi_{2}+\phi_{2}^{*}\right)^{2} .
$$

Or, in terms of the original bulk fields,

$$
\mathcal{L}_{5}{ }^{\prime}=\mathcal{L}_{5}+\partial_{5}\left(-\Phi D-\frac{1}{2}\left(\lambda_{1} \lambda_{2}+\text { h.c. }\right)\right)+\frac{1}{4} \partial_{m} \partial^{m}\left(\Phi^{2}\right) .
$$

In the orbifold picture both total derivatives can be neglected. But, as we will see in the next

section, in the boundary picture the total $\partial_{5}$ derivative gives rise to an important boundary term.

The brane action can also be written in the superfield form. The following Lagrangian,

$$
\mathcal{L}_{4}{ }^{\prime}=\int d^{2} \theta d^{2} \bar{\theta} \boldsymbol{\Phi}^{\dagger} e^{\mathbf{V}} \mathbf{\Phi}
$$


differs from $\mathcal{L}_{4}$, Eq. (2.18), by a total $\partial_{m}$ derivative,

$$
\begin{aligned}
\mathcal{L}_{4}{ }^{\prime} & =\mathcal{L}_{4}^{r}+\frac{1}{4} \partial_{m} \partial^{m}\left(\phi \phi^{*}\right) \\
& =\mathcal{L}_{4}+\frac{1}{4} \partial_{m} \partial^{m}\left(\phi \phi^{*}\right)-\frac{i}{2} \partial_{m}\left(\psi \sigma^{m} \bar{\psi}\right) .
\end{aligned}
$$

Therefore they both lead to the same brane action.

It is clear that using the $D=4, N=1$ superfields keeps the $N=1\left(\eta=\eta_{1}\right)$ supersymmetry manifest. A less obvious observation is that under the $N=1$ supersymmetry, the bulk Lagrangian $\mathcal{L}_{5}{ }^{\prime}$ varies into a total $\partial_{m}$ (not $\partial_{M}$ ) derivative term, that is

$$
\delta_{\eta} \mathcal{L}_{5}{ }^{\prime} \text { does not contain a } \partial_{5} \text { term. }
$$

(This is so because for the $D=4, N=1$ superfields $x^{5}$ is just a parameter. The highest component of a superfield varies into a total derivative, which is $\partial_{m}$ for our superfields.) This ensures that $\mathcal{L}_{5}{ }^{\prime}$ automatically gives rise to a supersymmetric action both in the orbifold and boundary pictures!

\subsection{Boundary conditions in the superfield form}

In the orbifold picture, the (superfield) bulk-plus-brane Lagrangian is

$$
\begin{aligned}
\mathcal{L}^{\prime}=\mathcal{L}_{5}{ }^{\prime}+\mathcal{L}_{4}{ }^{\prime} \delta(z)= & \frac{1}{4} \int d^{2} \theta \mathbf{W} \mathbf{W}+\text { h.c. } \\
& +\int d^{2} \theta d^{2} \bar{\theta}\left\{\mathbf{Z}^{2}+\boldsymbol{\Phi}^{\dagger} e^{\mathbf{V}} \mathbf{\Phi} \delta(z)\right\},
\end{aligned}
$$

where $\mathbf{Z}=\partial_{5} \mathbf{V}-\frac{1}{2}\left(\boldsymbol{\Phi}_{\mathbf{2}}+\boldsymbol{\Phi}_{\mathbf{2}}{ }^{\dagger}\right)$. One can derive the equations of motion directly from varying the superfields [24]. The variation of chiral superfields requires some care. But in our case all boundary conditions come from varying the vector superfield V. Keeping only terms with $\partial_{5}$ and $\delta(z)$, we obtain,

$$
\delta \mathcal{L}^{\prime}=\int d^{2} \theta d^{2} \bar{\theta}\left\{\delta \mathbf{V}\left[-2 \partial_{5} \mathbf{Z}+\boldsymbol{\Phi}^{\dagger} e^{\mathbf{V}} \mathbf{\Phi} \delta(z)\right]+\partial_{5}[2 \mathbf{Z} \delta \mathbf{V}]\right\}+\ldots
$$

The total $\partial_{5}$ derivative is irrelevant in the orbifold picture. (But, as we will see, it is essential in the derivation of boundary conditions in the boundary picture.) From the equation of motion,

$$
2 \partial_{5} \mathbf{Z}=\boldsymbol{\Phi}^{\dagger} e^{\mathbf{V}} \mathbf{\Phi} \delta(z)+\ldots
$$

and assuming the parity assignments (3.4) (so that $\mathbf{V}$ is even, while $\mathbf{\Phi}_{\mathbf{2}}$ and $\mathbf{Z}$ are odd), we obtain the following boundary condition,

$$
2 \mathbf{Z} \equiv 2 \partial_{5} \mathbf{V}-\left(\boldsymbol{\Phi}_{\mathbf{2}}+\boldsymbol{\Phi}_{\mathbf{2}}^{\dagger}\right) \stackrel{+0}{=} \frac{1}{2} \boldsymbol{\Phi}^{\dagger} e^{\mathbf{V}} \boldsymbol{\Phi}
$$


Using the component expansions (see Appendix B.2), we can split this superfield boundary condition into the following relations for the component fields,

$$
\begin{aligned}
& 1 \quad: \quad-\left(\phi_{2}+\phi_{2}^{*}\right) \stackrel{+0}{=} \frac{1}{2} \phi \phi^{*} \\
& \theta \quad: \quad-\sqrt{2} \psi_{2} \stackrel{+0}{=} \frac{1}{\sqrt{2}} \phi^{*} \psi \\
& \theta^{2} \quad: \quad-F_{2} \stackrel{+0}{=} \frac{1}{2} \phi^{*} F \\
& \theta \sigma^{m} \bar{\theta}:-2 \partial_{5} v_{m}-i\left(\partial_{m} \phi_{2}-\partial_{m} \phi_{2}^{*}\right) \stackrel{+0}{=}-\frac{i}{2}\left(\phi \mathcal{D}_{m} \phi^{*}-\phi^{*} \mathcal{D}_{m} \phi\right)-\frac{1}{2} \psi \sigma_{m} \bar{\psi} \\
& \bar{\theta}^{2} \theta \quad: \quad-2 i \partial_{5} \lambda-\frac{i}{\sqrt{2}} \sigma^{m} \partial_{m} \bar{\psi}_{2} \stackrel{+0}{=}-\frac{1}{2} \lambda \phi \phi^{*}+\frac{1}{\sqrt{2}} \psi F^{*} \\
& +\frac{i}{2 \sqrt{2}} \sigma^{m}\left(\phi \mathcal{D}_{m} \bar{\psi}-\bar{\psi} \mathcal{D}_{m} \phi\right) \\
& \theta^{2} \bar{\theta}^{2}: \quad \partial_{5} D-\frac{1}{4} \partial_{m} \partial^{m}\left(\phi_{2}+\phi_{2}^{*}\right) \stackrel{+0}{=} \frac{1}{2} \mathcal{L}_{4}^{r}+\frac{1}{8} \partial_{m} \partial^{m}\left(\phi \phi^{*}\right) .
\end{aligned}
$$

It is easy to check that these boundary conditions are equivalent to Eqs. (3.5) and (3.7). See also Appendix B.3.

Note that in the superfield approach all boundary conditions appear as primary boundary conditions and their supermultiplet structure is manifest.

The superfield derivation also explains how boundary conditions with and without $\partial_{5}$ can appear in the same supermultiplet. When $\mathbf{V}$ is not in the WZ gauge, $\partial_{5}$ appears in every boundary condition, but fixing the gauge allows one to eliminate terms with $\partial_{5}$ acting on the pure gauge degrees of freedom.

\section{Boundary picture}

In this section we will discuss supersymmetry and boundary conditions as they appear in the boundary picture, where our space-time domain is $\mathcal{M}=\mathbb{R}^{1,3} \times[0,+\infty)$. The space-time now has a boundary $\partial \mathcal{M}$ at $z=0$. We no longer have to deal with singularities, but now the total $\partial_{5}$ derivatives cannot be neglected. With our setting, we have

$$
\int_{\mathcal{M}} \partial_{M} K^{M}=\int_{\mathcal{M}} \partial_{5} K^{5}=\int_{\partial \mathcal{M}}\left(-K^{5}\right) \text {. }
$$

The measures of integration, $d^{5} x$ on $\mathcal{M}$ and $d^{4} x$ on $\partial \mathcal{M}$, are implicit.

\subsection{The action}

In the boundary picture, the bulk-plus-boundary action which leads to the same superfield boundary condition (4.11) is

$$
S=\int_{\mathcal{M}} \mathcal{L}_{5}{ }^{\prime}+\frac{1}{2} \int_{\partial \mathcal{M}} \mathcal{L}_{4}{ }^{\prime}
$$


(We can replace here $\mathcal{L}_{4}{ }^{\prime}$ by $\mathcal{L}_{4}$ since they differ only by a total $\partial_{m}$ derivative, Eq. (4.7), which integrates to zero on $\partial \mathcal{M}$.) Indeed, a general variation of the vector superfield $\mathbf{V}$ gives the following boundary term,

$$
\delta S=\int_{\partial \mathcal{M}} \int d^{2} \theta d^{2} \bar{\theta} \delta \mathbf{V}\left(-2 \mathbf{Z}+\frac{1}{2} \boldsymbol{\Phi}^{\dagger} e^{\mathbf{V}} \mathbf{\Phi}\right) .
$$

Requiring this term to vanish for arbitrary $\delta \mathbf{V}$, enforces the boundary condition (4.11),

$$
2 \mathbf{Z} \stackrel{+0}{=} \frac{1}{2} \boldsymbol{\Phi}^{\dagger} e^{\mathbf{V}} \boldsymbol{\Phi}
$$

\subsection{Important boundary term}

The two bulk Lagrangians, $\mathcal{L}_{5}$ and $\mathcal{L}_{5}{ }^{\prime}$, defined in Eqs. (2.1) and (4.2), respectively, lead to two different actions,

$$
S_{5}=\int_{\mathcal{M}} \mathcal{L}_{5} \text { and } S_{5}{ }^{\prime}=\int_{\mathcal{M}} \mathcal{L}_{5}{ }^{\prime}
$$

because of the $\partial_{5}$ term in Eq. (4.5). The actions differ by a boundary term. Namely,

$$
S_{5}{ }^{\prime}=S_{5}+\int_{\partial \mathcal{M}}\left(\Phi D+\frac{1}{2}\left(\lambda_{1} \lambda_{2}+\text { h.c. }\right)\right) .
$$

We know that $S_{5}{ }^{\prime}$ is supersymmetric, because $\mathcal{L}_{5}{ }^{\prime}$ was constructed out of $(D=4, N=1)$ superfields (see the last remark in Section 4.1). On the other hand, the action $S_{5}$, based on the original bulk Lagrangian (2.1), is not supersymmetric in the boundary picture. The extra boundary term is required to make the bulk action supersymmetric.

\subsection{Supersymmetry of the action}

It is instructive to check supersymmetry of the action $S_{5}{ }^{\prime}$ explicitly. We have

$$
\delta_{\eta} S_{5}^{\prime}=\int_{\partial \mathcal{M}}\left(-\widetilde{K}^{5}+\delta_{\eta}(\Phi D)+\frac{1}{2}\left(\delta_{\eta}\left(\lambda_{1} \lambda_{2}\right)+\text { h.c. }\right)\right),
$$

where $\widetilde{K}^{M}$ is given in Eq. (A.4). The boundary term can be rewritten as

$$
F^{5 n} \delta_{\eta} A_{n}+\Phi \delta_{\eta} D+X_{3} \delta_{\eta} \Phi+\left(\lambda_{2} \delta_{\eta}^{\prime \prime} \lambda_{1}+\lambda_{1} \delta_{\eta}^{\prime} \lambda_{2}+\text { h.c. }\right)
$$

where $\delta_{\eta}^{\prime \prime} \lambda_{1}=i X_{3} \eta$ and $\delta_{\eta}^{\prime} \lambda_{2}=-\left(i F_{m 5}+\partial_{m} \Phi\right) \sigma^{m} \bar{\eta}$. It is easy to check that this boundary term is a total $\partial_{m}$ derivative. This explicitly shows that the action $S_{5}{ }^{\prime}$ is supersymmetric,

$$
\delta_{\eta} S_{5}{ }^{\prime}=0
$$

We conclude that the total bulk-plus-boundary action (5.2),

$$
S=\int_{\mathcal{M}} \mathcal{L}_{5}+\int_{\partial \mathcal{M}} Y+\frac{1}{2} \int_{\partial \mathcal{M}} \mathcal{L}_{4}
$$


where

$$
Y=\Phi D+\frac{1}{2}\left(\lambda_{1} \lambda_{2}+\text { h.c. }\right)
$$

is $N=1$ supersymmetric. This statement does not rely on using any boundary conditions. The "improved" bulk action $S_{5}{ }^{\prime}$ (the sum of terms with $\mathcal{L}_{5}$ and $Y$ ), and the boundary action (with $\mathcal{L}_{4}$ ) are separately supersymmetric.

\subsection{Variational principle}

As we will now show, the boundary $Y$-term plays the role of the Gibbons-Hawking term for our bulk action. (We chose letter $Y$ to honor York [12, 13], whose name could as well be included in the phrase "Gibbons-Hawking term".)

Using Eq. (A.1), we find that the general variation of the original bulk action $S_{5}$ has the following boundary term,

$$
\delta S_{5}=\int_{\partial \mathcal{M}}\left(-K^{5}\right)=\int_{\partial \mathcal{M}}\left(\delta A_{n} F^{5 n}+\delta \Phi \partial_{5} \Phi+\frac{1}{2}\left(\lambda_{2} \delta \lambda_{1}-\lambda_{1} \delta \lambda_{2}+\text { h.c. }\right)\right) .
$$

This expression, however, gets modified when we make the following field redefinition,

$$
X_{3} \quad \longrightarrow \quad D=X_{3}-\partial_{5} \Phi
$$

Considering $D$ (rather than $X_{3}$ ) as an independent bulk field, we find

$$
\delta S_{5}=\int_{\partial \mathcal{M}}\left(\delta A_{n} F^{5 n}-\delta \Phi D+\frac{1}{2}\left(\lambda_{2} \delta \lambda_{1}-\lambda_{1} \delta \lambda_{2}+\text { h.c. }\right)\right) .
$$

The analogous general variation for the "improved" action (5.6) is

$$
\delta S_{5}{ }^{\prime}=\int_{\partial \mathcal{M}}\left(\delta A_{n} F^{5 n}+\Phi \delta D+\left(\lambda_{2} \delta \lambda_{1}+\text { h.c. }\right)\right) .
$$

This expression contains variations of only those (combinations of the) bulk fields which we include in the boundary action. (Just as the gravitational action with the GibbonsHawking boundary term [11] contains only variations of the metric field on the boundary, but not variations of its normal derivative.) Adding the contribution from the variation of the boundary action $\int_{\partial \mathcal{M}} \mathcal{L}_{4}$ and requiring the total expression to vanish for arbitrary variations $\delta A_{m}, \delta D$ and $\delta \lambda_{1}$, we obtain the primary boundary conditions (3.5). ${ }^{3}$

The boundary $Y$-term, therefore, plays two roles at the same time:

1) It makes the "improved" bulk action $S_{5}{ }^{\prime}$ supersymmetric.

2) It makes the variational principle well-defined (the equations of motion and the boundary conditions follow from the vanishing of the general variation of the action for arbitrary field variations).

\footnotetext{
${ }^{3}$ For comparison, see the derivation and discussion of boundary conditions in the boundary ("interval") picture in Refs. 20, 21.
} 


\section{On-shell case}

This section is of particular importance for the discussion of (on-shell) five-dimensional supergravity on a manifold with boundary given in the companion paper, Ref. [9].

In this section we will show that after eliminating the auxiliary fields only the total bulk-plus-brane action is supersymmetric (and not the bulk and the brane actions separately, as is the case in the off-shell formulation). In addition, in the on-shell boundary picture, supersymmetry of the total action does rely on using (some of) the boundary conditions!

\subsection{On-shell in the orbifold picture}

The total bulk-plus-brane Lagrangian is

$$
\mathcal{L}=\mathcal{L}_{5}+\delta(z) \mathcal{L}_{4}
$$

where $\mathcal{L}_{5}$ and $\mathcal{L}_{4}$ are given in Eqs. (2.1) and (2.18), respectively. The bulk fields $X_{1}, X_{2}$, $X_{3}$ and the brane field $F$ are auxiliary. Their equations of motion (see Eq. (A.30)) are pure algebraic (contain no derivatives) and can be used to set these fields to their on-shell values (denoted by the "breve" accent or the superscript "on"),

$$
\begin{aligned}
& \breve{X}_{1}=0, \quad \breve{X}_{2}=0, \quad \breve{F}=0 \\
& \breve{X}_{3}=-\frac{1}{2} \phi \phi^{*} \delta(z) .
\end{aligned}
$$

We would like to see if the on-shell expressions for $\mathcal{L}_{5}$ and $\mathcal{L}_{4}$ are supersymmetric. Instead of performing an explicit check, we use a short-cut.

Let us separate out terms containing the auxiliary fields,

$$
\begin{aligned}
& \mathcal{L}_{5}=\widehat{\mathcal{L}}_{5}+\frac{1}{2}\left(X_{1}^{2}+X_{2}^{2}+X_{3}^{2}\right) \\
& \mathcal{L}_{4}=\widehat{\mathcal{L}}_{4}+F F^{*}+\frac{1}{2} \phi \phi^{*} X_{3} .
\end{aligned}
$$

The supersymmetry variation of the hatted quantities (containing no auxiliary fields) commutes with setting the auxiliary fields to their on-shell values. Noting also that only $\breve{X}_{3}$ is non-zero, we obtain,

$$
\begin{aligned}
& \delta_{\eta}\left(\mathcal{L}_{5}^{\text {on }}\right)-\left(\delta_{\eta} \mathcal{L}_{5}\right)^{\text {on }}=\breve{X}_{3}\left[\delta_{\eta} \breve{X}_{3}-\left(\delta_{\eta} X_{3}\right)^{\text {on }}\right] \\
& \delta_{\eta}\left(\mathcal{L}_{4}^{\text {on }}\right)-\left(\delta_{\eta} \mathcal{L}_{4}\right)^{\text {on }}=\frac{1}{2} \phi \phi^{*}\left[\delta_{\eta} \breve{X}_{3}-\left(\delta_{\eta} X_{3}\right)^{\text {on }}\right] .
\end{aligned}
$$

We know that

$$
\delta_{\eta} \mathcal{L}_{5}=0, \quad \delta_{\eta} \mathcal{L}_{4}=0
$$

omitting total $\partial_{m}$ and $\partial_{5}$ derivatives, which are both irrelevant in the orbifold picture. The expression in the square brackets is proportional to the $\lambda_{1}$ equation of motion (see Eq. (A.30),

$$
\delta_{\eta} \breve{X}_{3}-\left(\delta_{\eta} X_{3}\right)^{\text {on }}=\eta_{1}\left\{i \partial_{5} \lambda_{2}+\sigma^{m} \partial_{m} \bar{\lambda}_{1}-\frac{1}{\sqrt{2}} \phi^{*} \psi \delta(z)\right\}+\text { h.c. }
$$


Since we are not allowed to use the equations of motion in checking supersymmetry, we conclude that the on-shell Lagrangians are not (separately) supersymmetric,

$$
\delta_{\eta}\left(\mathcal{L}_{5}^{\text {on }}\right) \neq 0, \quad \delta_{\eta}\left(\mathcal{L}_{4}^{\text {on }}\right) \neq 0 .
$$

On the other hand, for the total bulk-plus-brane Lagrangian we have

$$
\delta_{\eta}\left(\mathcal{L}^{\mathrm{on}}\right)-\left(\delta_{\eta} \mathcal{L}\right)^{\mathrm{on}}=\left(\breve{X}_{3}+\frac{1}{2} \phi \phi^{*} \delta(z)\right)\left[\delta_{\eta} \breve{X}_{3}-\left(\delta_{\eta} X_{3}\right)^{\text {on }}\right]
$$

which does vanish due to Eq. (6.3). Therefore, the total Lagrangian is supersymmetric,

$$
\delta_{\eta}\left(\mathcal{L}^{\text {on }}\right)=0
$$

and this does not rely on using any boundary conditions.

\subsection{On-shell in the boundary picture}

The total bulk-plus-boundary action is

$$
S=\int_{\mathcal{M}} \mathcal{L}_{5}{ }^{\prime}+\frac{1}{2} \int_{\partial \mathcal{M}} \mathcal{L}_{4}{ }^{\prime}
$$

Omitting total $\partial_{m}$ derivatives, but keeping total $\partial_{5}$ derivatives (essential in the boundary picture), we have,

$$
\mathcal{L}_{5}{ }^{\prime}=\mathcal{L}_{5}+\partial_{5}\left(-\Phi D-\frac{1}{2}\left(\lambda_{1} \lambda_{2}+\text { h.c. }\right)\right), \quad \mathcal{L}_{4}{ }^{\prime}=\mathcal{L}_{4}
$$

There is only a slight modification to the expressions obtained in the previous subsection,

$$
\begin{aligned}
& \delta_{\eta}\left(\mathcal{L}_{5}{ }^{\text {on }}\right)-\left(\delta_{\eta} \mathcal{L}_{5}{ }^{\prime}{ }^{\text {on }}=\breve{X}_{3}\left[\delta_{\eta} \breve{X}_{3}-\left(\delta_{\eta} X_{3}\right)^{\text {on }}\right]-\partial_{5}\left(\Phi\left[\delta_{\eta} \breve{X}_{3}-\left(\delta_{\eta} X_{3}\right)^{\text {on }}\right]\right)\right. \\
& \delta_{\eta}\left(\mathcal{L}_{4}{ }^{\text {on }}\right)-\left(\delta_{\eta} \mathcal{L}_{4}{ }^{\prime}\right)^{\text {on }}=\frac{1}{2} \phi \phi^{*}\left[\delta_{\eta} \breve{X}_{3}-\left(\delta_{\eta} X_{3}\right)^{\text {on }}\right] .
\end{aligned}
$$

But in the boundary picture, instead of a single auxiliary field equation (6.3), the variational principle for arbitrary $\delta X_{3}$ in the bulk and on the boundary produces a bulk equation of motion and a boundary condition,

$$
\breve{X}_{3}=0 \quad \oplus \stackrel{+0}{=}-\frac{1}{4} \phi \phi^{*}
$$

This is so because the terms involving $X_{3}$ in the bulk-plus-boundary action are

$$
S=\int_{\mathcal{M}} \frac{1}{2} X_{3}^{2}+\int_{\partial \mathcal{M}}\left(X_{3}-\partial_{5} \Phi\right)\left(\Phi+\frac{1}{4} \phi \phi^{*}\right)+\ldots
$$

We also know that

$$
\delta_{\eta} \mathcal{L}_{5}{ }^{\prime}=0, \quad \delta_{\eta} \mathcal{L}_{4}{ }^{\prime}=0
$$


(now omitting only total $\partial_{m}$ derivatives). Therefore, we find

$$
\begin{aligned}
& \delta_{\eta}\left(\mathcal{L}_{5}{ }^{\text {on }}\right)=\partial_{5}\left[\Phi\left(\delta_{\eta} X_{3}\right)^{\mathrm{on}}\right] \\
& \delta_{\eta}\left(\mathcal{L}_{4}{ }^{\text {on }}\right)=-\frac{1}{2} \phi \phi^{*}\left(\delta_{\eta} X_{3}\right)^{\mathrm{on}} .
\end{aligned}
$$

Combining these expressions, we obtain

$$
\delta_{\eta}\left(S^{\mathrm{on}}\right)=\int_{\partial \mathcal{M}}\left\{-\left(\Phi+\frac{1}{4} \phi \phi^{*}\right)\left(\delta_{\eta} X_{3}\right)^{\text {on }}\right\} .
$$

Therefore, the total bulk-plus-boundary action, restricted to on-shell field content, is supersymmetric only if we use the boundary condition $B_{1}(\Phi)$, Eq. (3.5),

$$
2 \Phi \stackrel{+0}{=}-\frac{1}{2} \phi \phi^{*} \quad \Longrightarrow \quad \delta_{\eta}\left(S^{\text {on }}\right)=0 .
$$

But this is exactly the boundary condition which comes as a part of the auxiliary field equation for $X_{3}$, Eq. (6.17). Thus, using this ("auxiliary") boundary condition is just a part of the going on-shell procedure!

Note that in the boundary picture the boundary condition $B_{1}(\Phi)$ also comes as a factor with the general variation $\delta D$ (and thus could also be called $B_{1}(D)$ ), and that on-shell $D=$ $-\partial_{5} \Phi$. Variations of $\Phi$ and $\partial_{5} \Phi$ on the boundary are independent. Our Gibbons-Hawking-like $Y$-term makes only the variation of $\partial_{5} \Phi$ appear on the boundary.

\subsection{On-shell closure of the supersymmetry algebra}

It is well-known that on-shell the supersymmetry algebra closes only up to equations of motion. This is true in our case as well, as one can explicitly check. But one should remember that in the orbifold picture the equations of motion contain $\delta(z)$ singularities. And this brings about one important issue.

Among all on-shell fields, only $\lambda_{1}$ has a singular term in its on-shell supersymmetry transformation. Indeed, off-shell we have

$$
\delta_{\eta} \lambda_{1}=\left(\sigma^{m n} F_{m n}-i \partial_{5} \Phi+i X_{3}\right) \eta_{1}
$$

which on-shell (in the orbifold picture) becomes

$$
\delta_{\eta} \lambda_{1}=\sigma^{m n} \eta_{1} F_{m n}-i\left[\partial_{5} \Phi+\frac{1}{2} \phi \phi^{*} \delta(z)\right] \eta_{1} .
$$

The commutator of two supersymmetry transformations on $\lambda_{1}$ gives

$$
\left[\delta_{\xi}, \delta_{\eta}\right] \lambda_{1}=-2 i U_{\xi \eta}^{m} \partial_{m} \lambda_{1}+\frac{1}{2} U_{\xi \eta}^{m}\left(\sigma_{m} \overline{E\left[\lambda_{1}\right]}\right)-\left(\xi \sigma^{m n} \eta\right)\left(\sigma_{m n} E\left[\lambda_{1}\right]\right)
$$

where $U_{\xi \eta}^{m}=\xi \sigma^{m} \bar{\eta}-\eta \sigma^{m} \bar{\xi}$, and

$$
E\left[\lambda_{1}\right] \equiv-i \sigma^{m} \partial_{m} \bar{\lambda}_{1}+\partial_{5} \lambda_{2}+\frac{i}{\sqrt{2}} \phi^{*} \psi \delta(z)=0
$$


is the equation of motion which comes with $\delta \lambda_{1}$, see Eq. (A.30). Note that without the singular term in $\delta_{\eta} \lambda_{1}$, the commutator closes up to the non-singular equation of motion.

We can make the following observations regarding the singular term

$$
\delta^{\prime} \lambda_{1}=-\frac{i}{2} \phi \phi^{*} \eta_{1} \delta(z):
$$

1) It comes from eliminating the auxiliary field $X_{3}$.

2) It makes the on-shell supersymmetry algebra close up to the full (singular) equations of motion.

3) It makes $\delta_{\eta} \lambda_{1}$ non-singular when the boundary condition $B_{1}(\Phi)$ (which fixes the jump of $\Phi$ across the brane) is taken into account.

The last observation provides a "rule of thumb" procedure for the appropriate modification of the supersymmetry transformations in the orbifold picture:

1. Identify all terms with $\partial_{5}$ acting on the odd fields.

2. Find the corresponding natural boundary conditions.

3. Add singular terms that make the modified supersymmetry transformation non-singular when the boundary conditions are taken into account.

This approach was already used in Ref. [8].

\subsection{The full square structure}

The on-shell Lagrangian in the orbifold picture contains a $\delta(z)^{2}$ term which comes from eliminating the $X_{3}$ auxiliary field. It turns out that this term combines with other terms into a full square. (This structure appears all the time in the orbifold constructions. It was first noticed by Horava [22]. We arrive to it also in Ref. [9].) Indeed,

$$
\begin{aligned}
\mathcal{L} & =\mathcal{L}_{5}+\mathcal{L}_{4} \delta(z) \\
& =-\frac{1}{2}\left(\partial_{5} \Phi\right)^{2}+\frac{1}{2} X_{3}^{2}+\frac{1}{2} \phi \phi^{*}\left(X_{3}-\partial_{5} \Phi\right) \delta(z)+\ldots \\
& =-\frac{1}{2}\left[\partial_{5} \Phi+\frac{1}{2} \phi \phi^{*} \delta(z)\right]^{2}+\ldots
\end{aligned}
$$

The complete on-shell Lagrangian in the orbifold picture can be written as follows,

$$
\begin{aligned}
\mathcal{L}= & -\frac{1}{4} F_{m n} F^{m n}-\frac{1}{2} \partial_{m} \Phi \partial^{m} \Phi-\left[\frac{i}{2} \lambda_{1} \sigma^{m} \partial_{m} \bar{\lambda}_{1}+\frac{i}{2} \lambda_{2} \sigma^{m} \partial_{m} \bar{\lambda}_{2}+\text { h.c. }\right] \\
& -\frac{1}{2} F_{m 5} F^{m 5}-\frac{1}{2}\left[\partial_{5} \Phi+\frac{1}{2} \phi \phi^{*} \delta(z)\right]^{2}+\left\{\lambda_{1}\left[\partial_{5} \lambda_{2}+\frac{i}{\sqrt{2}} \phi^{*} \phi \delta(z)\right]+\text { h.c. }\right\} \\
& -\left[\mathcal{D}_{m} \phi \mathcal{D}^{m} \phi^{*}+i \bar{\psi} \bar{\sigma}^{m} \mathcal{D}_{m} \psi\right] \delta(z) .
\end{aligned}
$$

We see that the combination $\partial_{5} \Phi+\frac{1}{2} \phi \phi^{*} \delta(z)$ appears both in the on-shell supersymmetry transformations and in the on-shell Lagrangian. The special property of this combination is that it is non-singular when the boundary condition for $\Phi$ is used. (Its analog in the five-dimensional supergravity [9] is $F_{m 5}+2 J_{m} \delta(z)$.) 


\subsection{On-shell closure of the boundary conditions}

Our natural boundary condition, Eq. 4.11), is

$$
2 \partial_{5} \mathbf{V}-\left(\boldsymbol{\Phi}_{\mathbf{2}}+\boldsymbol{\Phi}_{\mathbf{2}}^{\dagger}\right) \stackrel{+0}{=} \frac{1}{2} \boldsymbol{\Phi}^{\dagger} e^{\mathbf{V}} \boldsymbol{\Phi}
$$

In components, there are six boundary conditions: Eqs. (3.5) and (3.7). Off-shell they transform into each other under the $\left(\eta=\eta_{1}\right)$ supersymmetry transformations and thus form a closed orbit (see also Appendix B.3). Once we go on-shell, however, the boundary conditions are no longer closed under supersymmetry. Indeed, setting $F=0$ in $B_{2}\left(A_{m}\right)$ breaks the orbit, since $\delta F=0$ requires using the brane fermionic equation of motion (see Eqs. (A.24) and (A.30) ). Therefore, we need to use equations of motion to close the orbit of boundary conditions under supersymmetry on-shell (same as for the on-shell closure of the supersymmetry algebra!).

\subsection{The role of "odd $=0$ " boundary conditions}

When the bulk-brane coupling constant $Q$ is written explicitly (see footnote —), it multiplies the right-hand side of Eq. 6.31). Setting it to zero, gives the "odd =0" boundary conditions,

$$
2 \partial_{5} \mathbf{V}-\left(\boldsymbol{\Phi}_{\mathbf{2}}+\mathbf{\Phi}_{\mathbf{2}}^{\dagger}\right) \stackrel{+0}{=} 0
$$

which in components give

$$
F_{m 5} \stackrel{+0}{=} 0, \quad \Phi \stackrel{+0}{=} 0, \quad \lambda_{2} \stackrel{+0}{=} 0 \quad \partial_{5} \lambda_{1} \stackrel{+0}{=} 0, \quad X_{12} \stackrel{+0}{=} 0, \quad \partial_{5} D \stackrel{+0}{=} 0
$$

(It is interesting to note that they are closed under supersymmetry both off- and on-shell.) These boundary conditions were used in the original paper by Mirabelli and Peskin [2] as free field boundary conditions, for example, to derive the $k^{5}$ momentum quantization (see Eq. (26) in Ref. [2]). The coupling constant $Q$ (or its non-abelian analog $g t_{a b}^{A}$ ) is then reintroduced along the standard logic of the perturbation theory.

If one assumes, instead, that the "odd $=0$ " boundary conditions are the exact boundary conditions for the action with $Q \neq 0$, one would run into an inconsistency. These boundary conditions do not satisfy Eq. (4.11) and thus do not lead to the vanishing of the general variation of the action. It vanishes then only when the variations of the fields on the boundary are restricted to vanish themselves $(\delta \mathbf{V} \stackrel{+0}{=} 0$, "keeping $\mathbf{V}$ fixed on the boundary" à la Dirichlet boundary condition). But this implies fixing $\lambda_{1}$ and $\partial_{5} \lambda_{1}$ on the boundary at the same time, which gives an overdetermined boundary value problem! Also, since Eq. (6.23) does not hold, the on-shell bulk-plus-boundary action is not supersymmetric with these boundary conditions.

Therefore, the "odd $=0$ " boundary conditions can only be treated as the free field boundary conditions, that is as a starting point in the perturbative calculations. 


\section{From boundary to orbifold picture}

In order to better understand the role of the $Y$-term, Eq. (5.11), let us discuss the transition from the boundary to the orbifold picture. ${ }^{4}$

In the boundary picture we have the following total action,

$$
S^{(+)}=\int_{\mathcal{M}_{+}} \mathcal{L}_{5}+\int_{\partial \mathcal{M}_{+}} Y^{(+)}+\frac{1}{2} \int_{\partial \mathcal{M}_{+}} \mathcal{L}_{4}
$$

where $\mathcal{M}_{+}=\mathbb{R}^{1,3} \times[0,+\infty)$. The orbifold is, essentially, a union of two domains with boundary. The reflection of $\mathcal{M}_{+}$is $\mathcal{M}_{-}=\mathbb{R}^{1,3} \times(-\infty, 0]$. Its total bulk-plus-boundary action is

$$
S^{(-)}=\int_{\mathcal{M}_{-}} \mathcal{L}_{5}-\int_{\partial \mathcal{M}_{-}} Y^{(-)}+\frac{1}{2} \int_{\partial \mathcal{M}_{-}} \mathcal{L}_{4}
$$

The choice of signs is easy to understand. First, we have

$$
\int_{\mathcal{M}_{+}} \partial_{5} K^{5}=\int_{\partial \mathcal{M}_{+}}\left(-K^{5}\right), \quad \int_{\mathcal{M}_{-}} \partial_{5} K^{5}=\int_{\partial \mathcal{M}_{-}}\left(+K^{5}\right)
$$

which says that the signs of $Y^{(-)}$and $Y^{(+)}$relative to $\mathcal{L}_{5}$ should be opposite. Second, in the orbifold picture the $Y$-term is odd (since $\lambda_{2}$ and $\Phi$ are odd). Therefore, to get the correct boundary conditions, the signs of $Y^{(-)}$and $Y^{(+)}$relative to $\mathcal{L}_{4}$ should be opposite.

The boundaries of $\mathcal{M}_{+}$and $\mathcal{M}_{-}$coincide, so we denote

$$
\Sigma=\partial \mathcal{M}_{+}=\partial \mathcal{M}_{-} .
$$

Since the $Y$-term is odd, $Y^{(-)}=-Y^{(+)}$, the sum of the two bulk-plus-boundary actions is

$$
S=\int_{\mathcal{M}_{+} \cup \mathcal{M}_{-}} \mathcal{L}_{5}+\int_{\Sigma} 2 Y^{(+)}+\int_{\Sigma} \mathcal{L}_{4}
$$

We now want to show that this equals to our bulk-plus-brane action,

$$
S=\int_{\mathcal{M}_{5}} \mathcal{L}_{5}+\int_{\Sigma} \mathcal{L}_{4}=\int_{\mathcal{M}_{5}}\left\{\mathcal{L}_{5}+\mathcal{L}_{4} \delta(z)\right\}
$$

where $\mathcal{M}_{5}=\mathbb{R}^{1,4}=\mathbb{R}^{1,3} \times(-\infty,+\infty)$. This is equivalent to showing that the $Y$-term matches onto brane-localized terms produced by the bulk Lagrangian $\mathcal{L}_{5}$.

To do this, it helps to represent odd fields (in our case, $\Phi$ and $\lambda_{2}$ ) as follows,

$$
\Phi(x, z)=\varepsilon(z) \Phi^{(+)}(x,|z|),
$$

where $\varepsilon(z)=\operatorname{sgn}(z)= \pm 1$ on $\mathcal{M}_{ \pm}$. For the $\partial_{5}$ derivative of an odd field, we then have

$$
\partial_{5} \Phi=\varepsilon \partial_{5}\left[\Phi^{(+)}\right]+\Phi^{(+)} \varepsilon^{\prime}(z)=\left(\partial_{5} \Phi\right)^{(+)}+2 \Phi^{(+)} \delta(z) .
$$

\footnotetext{
${ }^{4}$ For an earlier discussion of the relationship between the orbifold and boundary pictures see, e.g., Ref. 23].
} 
(The superscript $(+)$ means "evaluated on the $\mathcal{M}_{+}$side".) This allows us to separate out the $\Sigma$-localized terms in $\mathcal{L}_{5}$.

The relevant terms in the bulk Lagrangian are

$$
\mathcal{L}_{5}=\frac{1}{2} X_{3}^{2}-\frac{1}{2}\left(\partial_{5} \Phi\right)^{2}+\frac{1}{2}\left(\lambda_{1} \partial_{5} \lambda_{2}+\text { h.c. }\right)+\ldots
$$

Using Eq. (7.8), we find

$$
\mathcal{L}_{5}=\frac{1}{2} X_{3}^{2}-2\left[\Phi^{(+)} \delta(z)\right]^{2}-2\left(\Phi \partial_{5} \Phi\right)^{(+)} \delta(z)+\left(\lambda_{1} \lambda_{2}^{(+)}+\text {h.c. }\right) \delta(z)+\ldots
$$

This is to be compared with

$$
2 Y^{(+)} \delta(z)=2\left[\Phi X_{3}\right]^{(+)} \delta(z)-2\left(\Phi \partial_{5} \Phi\right)^{(+)} \delta(z)+\left(\lambda_{1} \lambda_{2}^{(+)}+\text {h.c. }\right) \delta(z) .
$$

We see that terms without $X_{3}$ and $\delta(z)^{2}$ do match! The remaining terms appear to match only on-shell. Indeed, on-shell we have

$$
X_{3}=-\frac{1}{2} \phi \phi^{*} \delta(z), \quad X_{3}^{(+)}=0, \quad 2 \Phi^{(+)}=-\frac{1}{2} \phi \phi^{*}
$$

(including the "auxiliary boundary condition," Eq. (6.17)), which implies

$$
\frac{1}{2} X_{3}^{2}-2\left[\Phi^{(+)} \delta(z)\right]^{2}=0, \quad\left[\Phi X_{3}\right]^{(+)}=0 .
$$

We conclude, therefore, that the $Y$-term matches onto regular singularities (just $\delta(z)$ ) of the bulk Lagrangian, whereas higher order singularities (in our case $\delta(z)^{2}$ ) are taken care of by the auxiliary fields after going on-shell. In other words, only the on-shell part of the $Y$-term can be derived from the comparison with the brane-localized terms produced by the bulk Lagrangian.

\section{Summary and Conclusions}

In this paper we discussed the Mirabelli and Peskin model [2] in various settings: in the orbifold and boundary pictures, ${ }^{5}$ in components and in the superfield formulation, off-shell and on-shell.

We showed that the boundary picture requires introduction of the $Y$-term (GibbonsHawking-like term), which is necessary for supersymmetry and allows us to derive natural (Neumann-like) boundary conditions via the standard application of the variational principle. We found that the $Y$-term arises naturally in the $(D=4, N=1)$ superfield formulation of the $(D=5, N=2)$ model.

\footnotetext{
${ }^{5}$ The physics described by both pictures is guaranteed to be the same since the boundary conditions on the fundamental domain are identical. One advantage of the boundary picture is a complete removal of all ambiguities related to products of distributions.
} 
We demonstrated that, in the orbifold picture, $(N=1)$ supersymmetry does not require the use of any boundary conditions both off- and on-shell. In the boundary picture, however, supersymmetry of the total action requires the use of one boundary condition: the "auxiliary boundary condition," Eq. (6.17) (the one which comes as a part of the auxiliary equations of motion). This boundary condition is also one of the natural boundary conditions.

We showed that the natural boundary conditions form a closed orbit under $(N=1)$ supersymmetry and can be put in a superfield form. We can identify the "boundary condition superfield" (see Appendix B.3). The natural boundary conditions reduce to the "odd=0" boundary conditions only in the absence of coupling to the brane-localized matter.

We also saw what modifications to the supersymmetry transformations are necessary in the orbifold picture, and confirmed that the $\delta(z)^{2}$ terms fit into the full square structure in the Lagrangian. We found that the $Y$-term matches onto regular singularities of the bulk Lagrangian, but that higher order singularities (like $\left.\delta(z)^{2}\right)$ are taken care of only on-shell.

The detailed analysis of this simple model serves as a basis for the analysis in Ref. [9], where we discuss five-dimensional (on-shell) supergravity on a manifold with boundary.

\section{Acknowledgments}

I would like to thank Jonathan Bagger for helpful discussions and critical reading of this manuscript. I would also like to thank Fabio Zwirner for his questions on the closure of boundary conditions in Ref. [8] under supersymmetry, which were a part of the motivation for this work. This work was supported in part by the National Science Foundation, grant NSF-PHY-0401513.

\section{A. Details of the component formulation}

Here we collect various technical details, which were only briefly mentioned in the body of the paper. The basic conventions are as in Ref. [8].

\section{A.1 Variation of the bulk Lagrangian}

Under a general variation of the fields, the bulk Lagrangian varies as follows,

$$
\begin{aligned}
\delta \mathcal{L}_{5}= & \delta A_{N}\left(\partial_{M} F^{M N}\right)+\delta \Phi\left(\partial_{M} \partial^{M} \Phi\right)+X_{a} \delta X_{a} \\
& -i \delta \widetilde{\Lambda}^{i} \Gamma^{M} \partial_{M} \Lambda_{i}+\partial_{M} K^{M},
\end{aligned}
$$

where

$$
K^{M}=-F^{M N} \delta A_{N}-\delta \Phi \partial^{M} \Phi-\frac{i}{2} \widetilde{\Lambda}^{i} \Gamma^{M} \delta \Lambda^{i}
$$

The variation of the Lagrangian under the supersymmetry transformations, Eq. (2.2), prefers a different separation of the total derivative term,

$$
\begin{aligned}
\delta_{\mathcal{H}} \mathcal{L}_{5}= & \delta_{\mathcal{H}} A_{N}\left(\partial_{M} F^{M N}\right)+\delta_{\mathcal{H}} \Phi\left(\partial_{M} \partial^{M} \Phi\right)+X_{a} \delta_{\mathcal{H}} X_{a} \\
& -i \widetilde{\Lambda}^{i} \Gamma^{M} \partial_{M} \delta_{\mathcal{H}}^{\prime} \Lambda_{i}-i \delta_{\mathcal{H}}^{\prime \prime} \widetilde{\Lambda}^{i} \Gamma^{M} \partial_{M} \Lambda_{i}+\partial_{M} \widetilde{K}^{M}
\end{aligned}
$$


where

$$
\widetilde{K}^{M}=-F^{M N} \delta_{\mathcal{H}} A_{N}-\delta_{\mathcal{H}} \Phi \partial^{M} \Phi-\frac{i}{2} \delta_{\mathcal{H}}^{\prime} \widetilde{\Lambda}^{i} \Gamma^{M} \Lambda_{i}-\frac{i}{2} \widetilde{\Lambda}^{i} \Gamma^{M} \delta_{\mathcal{H}}^{\prime \prime} \Lambda_{i}
$$

and we used the following split in the supersymmetry transformation of the gaugino,

$$
\begin{aligned}
\delta_{\mathcal{H}} \Lambda_{i}= & \delta_{\mathcal{H}}^{\prime} \Lambda_{i}+\delta_{\mathcal{H}}^{\prime \prime} \Lambda_{i} \\
& \delta_{\mathcal{H}}^{\prime} \Lambda_{i}=\left(\Sigma^{M N} F_{M N}+\Gamma^{M} \partial_{M} \Phi\right) \mathcal{H}_{i}, \quad \delta_{\mathcal{H}}^{\prime \prime} \lambda_{i}=X_{a}\left(\sigma_{a}\right)_{i}{ }^{j} \mathcal{H}_{j}
\end{aligned}
$$

It is easy to check that terms outside the total derivative cancel, thus giving

$$
\delta_{\mathcal{H}} \mathcal{L}_{5}=\partial_{M} \widetilde{K}^{M}
$$

\section{A.2 Two-component spinor notation}

Making the $M=\{m, 5\}$ split in the supersymmetry transformations and rewriting them in terms of the two-component Weyl spinors, we find

$$
\begin{aligned}
\delta_{\mathcal{H}} A^{m} & =i\left(\eta_{1} \sigma^{m} \bar{\lambda}_{1}+\eta_{2} \sigma^{m} \bar{\lambda}_{2}\right)+h . c . \\
\delta_{\mathcal{H}} A^{5} & =-\eta_{1} \lambda_{2}+\eta_{2} \lambda_{1}+h . c . \\
\delta_{\mathcal{H}} \Phi & =i\left(-\eta_{1} \lambda_{2}+\eta_{2} \lambda_{1}\right)+h . c . \\
\delta_{\mathcal{H}} \lambda_{1} & =\left(\sigma^{m n} F_{m n}-i \partial_{5} \Phi+i X_{3}\right) \eta_{1}+\left(i F_{m 5}+\partial_{m} \Phi\right) \sigma^{m} \bar{\eta}_{2}-i X_{12}^{*} \eta_{2} \\
\delta_{\mathcal{H}} \lambda_{2} & =-\left(i F_{m 5}+\partial_{m} \Phi\right) \sigma^{m} \bar{\eta}_{1}-i X_{12} \eta_{1}+\left(\sigma^{m n} F_{m n}-i \partial_{5} \Phi-i X_{3}\right) \eta_{2} \\
\delta_{\mathcal{H}} X_{12} & =2 \bar{\eta}_{1}\left(i \partial_{5} \bar{\lambda}_{1}-\bar{\sigma}^{m} \partial_{m} \lambda_{2}\right)+2 \eta_{2}\left(i \partial_{5} \lambda_{2}+\sigma^{m} \partial_{m} \bar{\lambda}_{1}\right) \\
\delta_{\mathcal{H}} X_{3} & =-\eta_{1}\left(i \partial_{5} \lambda_{2}+\sigma^{m} \partial_{m} \bar{\lambda}_{1}\right)-\eta_{2}\left(i \partial_{5} \lambda_{1}-\sigma^{m} \partial_{m} \bar{\lambda}_{2}\right)+\text { h.c. },
\end{aligned}
$$

where we defined $X_{12}=X_{1}+i X_{2}$. We used the following relation between the symplecticMajorana spinor $\Lambda_{i}$ and the pair $\left(\lambda_{1}, \lambda_{2}\right)$,

$$
\Lambda_{1}=-\Lambda^{2}=\left(\begin{array}{c}
\lambda_{1} \\
\bar{\lambda}_{2}
\end{array}\right), \quad \Lambda_{2}=\Lambda^{1}=\left(\begin{array}{c}
-\lambda_{2} \\
\bar{\lambda}_{1}
\end{array}\right) .
$$

The same relation holds between $\mathcal{H}_{i}$ and $\left(\eta_{1}, \eta_{2}\right)$. The Majorana conjugation, denoted by a tilde, gives

$$
\widetilde{\Lambda}_{1}=\left(-\lambda_{1}, \bar{\lambda}_{2}\right)
$$

\section{A.3 $S U(2)$ rotation}

The bulk Lagrangian is invariant (and the bulk supersymmetry transformations are covariant) under a global $S U(2)$ rotation,

$$
\Lambda_{i}^{\prime}=U_{i}^{j} \Lambda_{j}, \quad X_{a}{ }^{\prime} \sigma_{a}=U\left(X_{a} \sigma_{a}\right) U^{\dagger}
$$


where $U \in S U(2)$ is a constant matrix, and $\sigma_{a}$ are the Pauli matrices such that

$$
X_{a} \sigma_{a}=\left(\begin{array}{cc}
X_{3} & X_{12}^{*} \\
X_{12} & -X_{3}
\end{array}\right) \text {. }
$$

A particularly useful $S U(2)$ rotation is

$$
\lambda_{1}^{\prime}=\frac{\lambda_{1}-\alpha^{*} \lambda_{2}}{\sqrt{1+\alpha \alpha^{*}}}, \quad \lambda_{2}^{\prime}=\frac{\alpha \lambda_{1}+\lambda_{2}}{\sqrt{1+\alpha \alpha^{*}}}
$$

(same for $\left.\left(\eta_{1}, \eta_{2}\right)\right)$, accompanied by

$$
\begin{aligned}
X_{12}{ }^{\prime} & =\frac{X_{12}-\alpha^{2} X_{12}^{*}-2 \alpha X_{3}}{1+\alpha \alpha^{*}} \\
X_{3}{ }^{\prime} & =\frac{\alpha X_{12}^{*}+\alpha^{*} X_{12}+\left(1-\alpha \alpha^{*}\right) X_{3}}{1+\alpha \alpha^{*}} .
\end{aligned}
$$

The inverse transformation is obtained by changing the sign of $\alpha$. In particular,

$$
\eta_{2}=\frac{-\alpha \eta_{1}^{\prime}+\eta_{2}^{\prime}}{\sqrt{1+\alpha \alpha^{*}}}
$$

so that

$$
\eta_{2}=0 \quad \Rightarrow \quad \eta_{2}^{\prime}=\alpha \eta_{1}^{\prime}
$$

\section{A.4 $N=1$ supersymmetry and the gauge transformation}

Under $\eta=\eta_{1}$ supersymmetry, the five-dimensional gauge supermultiplet splits into two fourdimensional supermultiplets. These are a gauge multiplet (in the WZ gauge)

$$
v_{m}=A_{m}, \quad \lambda=\lambda_{1}, \quad D=X_{3}-\partial_{5} \Phi
$$

and a chiral multiplet

$$
\phi_{2}=\Phi+i A_{5}, \quad \psi_{2}=-i \sqrt{2} \lambda_{2}, \quad F_{2}=-X_{12} .
$$

The definitions lead to the standard transformation laws for the gauge multiplet,

$$
\begin{aligned}
& \delta_{\eta} v_{m}=i \eta \sigma_{m} \bar{\lambda}+\text { h.c. } \\
& \delta_{\eta} \lambda=\sigma^{m n} \eta v_{m n}+i \eta D \\
& \delta_{\eta} D=-\eta \sigma^{m} \partial_{m} \bar{\lambda}+\text { h.c. }
\end{aligned}
$$

as well as to the following supersymmetry transformations for the chiral multiplet,

$$
\begin{aligned}
\delta_{\eta} \phi_{2} & =\sqrt{2} \eta \psi_{2} \\
\delta_{\eta} \psi_{2} & =i \sqrt{2} \sigma^{m} \bar{\eta} \partial_{m} \phi_{2}+\sqrt{2} \eta F_{2}+\sqrt{2} \sigma^{m} \bar{\eta} \partial_{5} v_{m} \\
\delta_{\eta} F_{2} & =i \sqrt{2} \overline{\eta \sigma}^{m} \partial_{m} \psi_{2}-2 i \bar{\eta} \partial_{5} \bar{\lambda} .
\end{aligned}
$$


The latter differ from the standard transformations by extra terms involving $\partial_{5}$. One can check that the supersymmetry algebra closes up to a gauge transformation,

$$
\left[\delta_{\xi}, \delta_{\eta}\right]=-2 i U_{\xi \eta}^{m} \partial_{m}+\delta_{u}
$$

where $U_{\xi \eta}^{m}=\xi \sigma^{m} \bar{\eta}-\eta \sigma^{m} \bar{\xi}$ and the gauge transformation is non-zero only on $v_{m}$ and $\phi_{2}$,

$$
\delta_{u} v_{m}=\partial_{m} u, \quad \delta_{u} \phi_{2}=i \partial_{5} u,
$$

with $u=2 i U_{\xi \eta}^{m} v_{m}$. This is just the $U(1)$ gauge transformation $\delta A_{M}=\partial_{M} u$.

The chiral multiplet $(\phi, \psi, F)$, living on the brane, has the standard supersymmetry transformations (note the dependence on $v_{m}$ and $\lambda$ from the vector multiplet),

$$
\begin{aligned}
\delta_{\eta} \phi & =\sqrt{2} \eta \psi \\
\delta_{\eta} \psi & =i \sqrt{2} \sigma^{m} \bar{\eta} \mathcal{D}_{m} \phi+\sqrt{2} \eta F \\
\delta_{\eta} F & =i \sqrt{2} \bar{\eta}^{m} \mathcal{D}_{m} \psi+i \phi \bar{\eta} \bar{\lambda}
\end{aligned}
$$

where

$$
\mathcal{D}_{m}=\partial_{m}+\frac{i}{2} v_{m}
$$

when acting on $(\phi, \psi, F)$. This is the gauge covariant derivative, corresponding to the following $U(1)$ transformation of the chiral multiplet,

$$
\delta_{u}(\phi, \psi, F)=-\frac{i}{2} u(\phi, \psi, F) .
$$

The algebra (A.22) holds for this multiplet as well.

\section{A.5 Orbifold equations of motion}

In the orbifold picture the total Lagrangian is $\mathcal{L}=\mathcal{L}_{5}+\delta(z) \mathcal{L}_{4}$. For a general variation of the fields, we find

$$
\begin{aligned}
\delta \mathcal{L}_{5}= & \delta A_{n}\left(\partial_{m} F^{m n}+\partial_{5} F^{5 n}\right)+\delta A_{5}\left(\partial_{m} F^{m 5}\right)+\delta \Phi\left(\partial_{m} \partial^{m} \Phi+\partial_{5} \partial_{5} \Phi\right)+X_{a} \delta X_{a} \\
& -\left[\delta \lambda_{1}\left(i \sigma^{m} \partial_{m} \bar{\lambda}_{1}-\partial_{5} \lambda_{2}\right)+\delta \lambda_{2}\left(i \sigma^{m} \partial_{m} \bar{\lambda}_{2}+\partial_{5} \lambda_{1}\right)+h . c .\right] \\
\delta \mathcal{L}_{4}= & \delta \phi\left(\mathcal{D}_{m} \mathcal{D}^{m} \phi^{*}+\frac{1}{2} \phi^{*} D-\frac{i}{\sqrt{2}} \overline{\lambda \psi}\right)+\delta \psi\left(-i \sigma^{m} \mathcal{D}_{m} \bar{\psi}+\frac{i}{\sqrt{2}} \phi^{*} \lambda\right)+F^{*} \delta F \\
& +\delta v_{m}\left(-\frac{i}{2} \phi \mathcal{D}^{m} \phi^{*}-\frac{1}{4} \psi \sigma^{m} \bar{\psi}\right)+\delta \lambda\left(\frac{i}{\sqrt{2}} \phi^{*} \psi\right)+\delta D\left(\frac{1}{4} \phi \phi^{*}\right)+\text { h.c. }
\end{aligned}
$$

(Total $\partial_{m}$ and $\partial_{5}$ derivatives have been omitted here.) Using the definitions in Eq. (A.18) and rewriting

$$
\delta D \phi \phi^{*} \delta(z)=\delta X_{3} \phi \phi^{*} \delta(z)+\delta \Phi \partial_{5}\left(\phi \phi^{*} \delta(z)\right)
$$


we find the following equations of motion,

$$
\begin{array}{rlrl}
E\left(A_{m}\right) & : \partial_{n} F^{n m}-\partial_{5} F^{m 5}-\left(\frac{i}{2}\left(\phi \mathcal{D}^{m} \phi^{*}-\phi^{*} \mathcal{D}^{m} \phi\right)+\frac{1}{2} \psi \sigma^{m} \bar{\psi}\right) \delta(z) & =0 \\
E\left(A_{5}\right): & : & \partial_{m} F^{m 5} & =0 \\
E(\Phi) & : & \partial_{m} \partial^{m} \Phi+\partial_{5}\left(\partial_{5} \Phi+\frac{1}{2} \phi \phi^{*} \delta(z)\right) & =0 \\
E\left(\lambda_{1}\right): & -i \sigma^{m} \partial_{m} \bar{\lambda}+\partial_{5} \lambda_{2}+\frac{i}{\sqrt{2}} \phi^{*} \psi \delta(z) & =0 \\
E\left(\lambda_{2}\right): & -i \sigma^{m} \partial_{m} \bar{\lambda}_{2}-\partial_{5} \lambda & =0 \\
E\left(X_{12}\right): & X_{3}+\frac{1}{2} \phi \phi^{*} \delta(z) & =0 \\
E\left(X_{3}\right): & \mathcal{D}_{m} \mathcal{D}^{m} \phi^{*}+\frac{1}{2} \phi^{*} D-\frac{i}{\sqrt{2}} \overline{\lambda \psi} & =0 \\
E(\phi) & : & -i \sigma^{m} \mathcal{D}_{m} \bar{\psi}+\frac{i}{\sqrt{2}} \phi^{*} \lambda & =0 \\
E(\psi) & : & F^{*} & =0 . \\
E(F) & : &
\end{array}
$$

\section{B. Superfields}

We follow the conventions of Ref. [24]. See also Ref. [25].

\section{B.1 Supersymmetry and gauge transformations}

The vector and chiral superfields have the following component expansions,

$$
\begin{aligned}
& \mathbf{V}=-i \theta \sigma^{m} \bar{\theta} v_{m}+i \theta^{2} \overline{\theta \lambda}-i \bar{\theta}^{2} \theta \lambda+\frac{1}{2} \theta^{2} \bar{\theta}^{2} D \\
& \mathbf{\Phi}=\phi+i \theta \sigma^{m} \bar{\theta} \partial_{m} \phi+\frac{1}{4} \theta^{2} \bar{\theta}^{2} \partial_{m} \partial^{m} \phi+\sqrt{2} \theta \psi+\frac{i}{\sqrt{2}} \theta^{2} \bar{\theta} \bar{\sigma}^{m} \partial_{m} \psi+\theta^{2} F .
\end{aligned}
$$

The chiral superfields can be more conveniently written in terms of the " $y$ coordinates" $\left(y^{m}=x^{m}+\theta \sigma^{m} \bar{\theta}\right)$,

$$
\boldsymbol{\Phi}(y)=\phi(y)+\sqrt{2} \theta \psi(y)+\theta^{2} F(y) .
$$

The field strength $\mathbf{W}$ of the vector superfield $\mathbf{V}$ is a chiral spinor superfield (its lowest component is a spinor), which in the $y$-coordinates has the following form,

$$
\mathbf{W}(y)=-i \lambda+\theta D-i \sigma^{m n} \theta v_{m n}+\theta^{2}\left(\sigma^{m} \partial_{m} \bar{\lambda}\right),
$$

where $v_{m n}=\partial_{m} v_{n}-\partial_{n} v_{m}$. 
The supersymmetry transformations in Eqs. (A.20), (A.21) and (A.24) can be written in the following superfield form,

$$
\begin{aligned}
& \delta_{\eta} \mathbf{V}=(\eta Q+\bar{\eta} \bar{Q}) \mathbf{V}+\boldsymbol{\Phi}_{\eta}+\boldsymbol{\Phi}_{\eta}^{\dagger} \\
& \delta_{\eta} \mathbf{\Phi}=(\eta Q+\bar{\eta} \bar{Q}) \boldsymbol{\Phi}-\boldsymbol{\Phi}_{\eta} \boldsymbol{\Phi} \\
& \delta_{\eta} \boldsymbol{\Phi}_{\mathbf{2}}=(\eta Q+\bar{\eta} \bar{Q}) \boldsymbol{\Phi}_{\mathbf{2}}+2 \partial_{5} \boldsymbol{\Phi}_{\eta},
\end{aligned}
$$

where $\boldsymbol{\Phi}_{\eta}$ is a chiral superfield given by

$$
\mathbf{\Phi}_{\eta}(y)=\sqrt{2} \theta\left(\frac{1}{\sqrt{2}} \sigma^{m} \bar{\eta} v_{m}\right)+\theta^{2}(-i \bar{\eta} \bar{\lambda}) .
$$

It describes a compensating supergauge transformation necessary to keep $\mathbf{V}$ in the WZ gauge. From this we deduce that the supergauge transformation for all the superfields is given by

$$
\delta \mathbf{V}=\boldsymbol{\Lambda}+\boldsymbol{\Lambda}^{\dagger}, \quad \delta \Phi=-\boldsymbol{\Lambda} \Phi, \quad \delta \Phi_{2}=\mathbf{2} \partial_{\mathbf{5}} \boldsymbol{\Lambda} .
$$

The residual gauge transformation, preserving the WZ gauge, corresponds to

$$
-2 i \boldsymbol{\Lambda}=u(y)=u+i \theta \sigma^{m} \bar{\theta} \partial_{m} u+\frac{1}{2} \theta^{2} \bar{\theta}^{2} \partial_{m} \partial^{m} u .
$$

In components this gauge transformation reproduces Eqs. (A.23) and (A.26).

\section{B.2 Component expansions}

The component expansions for the bulk action are:

$$
\begin{array}{r}
\int d^{2} \theta \mathbf{W} \mathbf{W}+h . c .=-v_{m n} v^{m n}-2 i\left(\lambda \sigma^{m} \partial_{m} \bar{\lambda}+\bar{\lambda} \bar{\sigma}^{m} \partial_{m} \lambda\right)+2 D^{2} \\
\int d^{2} \theta d^{2} \bar{\theta} \mathbf{Z}^{2}=-\frac{1}{2} \partial_{m} \phi_{2} \partial^{m} \phi_{2}^{*}+\frac{1}{2} F_{2} F_{2}^{*}-\frac{i}{4}\left(\bar{\psi}_{2} \bar{\sigma}^{m} \partial_{m} \psi_{2}+\psi_{2} \sigma^{m} \partial_{m} \bar{\psi}_{2}\right) \\
-\frac{1}{2}\left(\partial_{5} v_{m}\right)\left(\partial_{5} v^{m}\right)-\frac{i}{2}\left(\partial_{m} \phi_{2}-\partial_{m} \phi_{2}^{*}\right) \partial_{5} v^{m}-\frac{1}{2}\left(\phi_{2}+\phi_{2}^{*}\right) \partial_{5} D \\
+\frac{i}{\sqrt{2}}\left(\bar{\psi}_{2} \partial_{5} \bar{\lambda}-\psi_{2} \partial_{5} \lambda\right)+\frac{1}{16} \partial^{m} \partial_{m}\left(\phi_{2}+\phi_{2}^{*}\right)^{2}
\end{array}
$$

The component expansions for the brane action and the boundary conditions are:

$$
\begin{aligned}
\partial_{5} \mathbf{V}= & -i \bar{\theta}^{2} \theta \partial_{5} \lambda+h . c .-i \theta \sigma^{m} \bar{\theta} \partial_{5} v_{m}+\frac{1}{2} \theta^{2} \bar{\theta}^{2} \partial_{5} D \\
\mathbf{\Phi}_{2}+\boldsymbol{\Phi}_{2}^{\dagger}= & \sqrt{2} \theta \psi_{2}+\theta^{2} F_{2}+\frac{i}{\sqrt{2}} \bar{\theta}^{2} \theta \sigma^{m} \partial_{m} \bar{\psi}_{2}+h . c . \\
& +\left(\phi_{2}+\phi_{2}^{*}\right)+i \theta \sigma^{m} \bar{\theta}\left(\partial_{m} \phi_{2}-\partial_{m} \phi_{2}^{*}\right)+\frac{1}{4} \theta^{2} \bar{\theta}^{2} \partial_{m} \partial^{m}\left(\phi_{2}+\phi_{2}^{*}\right) \\
\boldsymbol{\Phi}^{\dagger} e^{\mathbf{V}} \mathbf{\Phi}= & \sqrt{2} \theta\left(\phi^{*} \psi\right)+\theta^{2}\left(\phi^{*} F\right) \\
& +\bar{\theta}^{2} \theta\left(-i \lambda \phi \phi^{*}+\frac{i}{\sqrt{2}} \sigma^{m}\left(\mathcal{D}_{m} \bar{\psi} \phi-\bar{\psi} \mathcal{D}_{m} \phi\right)+\sqrt{2} \psi F^{*}\right)+h . c . \\
& +\left(\phi \phi^{*}\right)+\theta \sigma^{m} \bar{\theta}\left(-i\left(\phi \mathcal{D}_{m} \phi^{*}-\phi^{*} \mathcal{D}_{m} \phi\right)-\psi \sigma_{m} \bar{\psi}\right) \\
& +\theta^{2} \bar{\theta}^{2}\left(\mathcal{L}_{4}^{r}+\frac{1}{4} \partial_{m} \partial^{m}\left(\phi \phi^{*}\right)\right)
\end{aligned}
$$


where $\mathcal{L}_{4}^{r}$ is given in Eq. (3.8).

\section{B.3 Boundary condition superfield}

The boundary condition superfield is

$$
\mathbf{B}=2 \partial_{5} \mathbf{V}-\left(\boldsymbol{\Phi}_{\mathbf{2}}+\boldsymbol{\Phi}_{\mathbf{2}}^{\dagger}\right)-\frac{1}{2} \boldsymbol{\Phi}^{\dagger} e^{\mathbf{V}} \boldsymbol{\Phi} .
$$

Since $\mathbf{B}$ is a vector superfield, we can write 24]

$$
\begin{aligned}
\mathbf{B}= & i \theta \chi^{B}+\frac{i}{2} \theta^{2} M^{B}-i \theta^{2} \bar{\theta} \lambda^{B}+h . c . \\
& +C^{B}-i \theta \sigma^{m} \bar{\theta} v_{m}^{B}+\frac{1}{2} \theta^{2} \bar{\theta}^{2} D^{B}
\end{aligned}
$$

and identify each component as

$$
\begin{aligned}
C^{B} & =-\frac{1}{2} \phi \phi^{*}-2 \Phi \\
\chi^{B} & =\frac{i}{\sqrt{2}} \phi^{*} \phi+2 \lambda_{2} \\
M^{B} & =i\left(F \phi^{*}-2 X_{12}\right) \\
v_{m}^{B} & =-\frac{i}{2}\left(\phi D_{m} \phi^{*}-\phi^{*} D_{m} \phi\right)-\frac{1}{2} \phi \sigma_{m} \bar{\psi}-2 F_{m 5} \\
\lambda^{B} & =-\frac{i}{\sqrt{2}} \sigma^{m} \bar{\psi} D_{m} \phi+\frac{1}{\sqrt{2}} \psi F^{*}-\frac{i}{2} \phi \phi^{*} \lambda_{1}+2 i \partial_{5} \lambda_{1} \\
D^{B} & =-\mathcal{L}_{4}^{r}+2 \partial_{5} D .
\end{aligned}
$$

The relation to the boundary conditions, Eqs. (3.5) and (3.7), is as follows,

$$
\begin{array}{lll}
C^{B}: B_{1}(\Phi) & \chi^{B}: B_{1}\left(\lambda_{1}\right) & M^{B}: B_{2}\left(\lambda_{1}\right) \\
v_{m}^{B}: B_{1}\left(A_{m}\right) & \lambda^{B}: B_{2}\left(A_{m}\right) & D^{B}: B_{3}\left(A_{m}\right) .
\end{array}
$$

Using Eq. (B.5), we can easily show that

$$
\delta_{\eta} \mathbf{B}=(\eta Q+\bar{\eta} \bar{Q}) \mathbf{B},
$$

which in components gives (the superscript $B$ is omitted for clarity)

$$
\begin{aligned}
\delta_{\eta} C & =i \eta \chi+h . c . \\
\delta_{\eta} \chi & =\sigma^{m} \eta\left(\partial_{m} C+i v_{m}\right)+\eta M \\
\delta_{\eta} M & =2 \bar{\eta}\left(\bar{\lambda}+\bar{\sigma}^{m} \partial_{m} \chi\right) \\
\delta_{\eta} D & =-\eta \sigma^{m} \partial_{m} \bar{\lambda}+h . c . \\
\delta_{\eta} \lambda & =2 \sigma^{m n} \eta \partial_{m} v_{n}+i \eta D \\
\delta_{\eta} v_{m} & =i \eta \sigma_{m} \bar{\lambda}+\eta \partial_{m} \chi+h . c .
\end{aligned}
$$

This shows explicitly the structure of supersymmetry variations in the orbit of boundary conditions (cf. Eq. (3.6)). 


\section{References}

[1] P. Horava and E. Witten, "Eleven-Dimensional Supergravity on a Manifold with Boundary," Nucl. Phys. B 475, 94 (1996) [arXiv:hep-th/9603142].

[2] E. A. Mirabelli and M. E. Peskin, "Transmission of supersymmetry breaking from a 4-dimensional boundary," Phys. Rev. D 58, 065002 (1998) [arXiv:hep-th/9712214].

[3] L. Randall and R. Sundrum, "A large mass hierarchy from a small extra dimension," Phys. Rev. Lett. 83, 3370 (1999) [arXiv:hep-ph/9905221].

[4] L. Randall and R. Sundrum, "An alternative to compactification," Phys. Rev. Lett. 83, 4690 (1999) [arXiv:hep-th/9906064].

[5] R. Altendorfer, J. Bagger and D. Nemeschansky, "Supersymmetric Randall-Sundrum scenario," Phys. Rev. D 63, 125025 (2001) [hep-th/0003117].

[6] T. Gherghetta and A. Pomarol, "Bulk fields and supersymmetry in a slice of AdS," Nucl. Phys. B 586, 141 (2000) [hep-ph/0003129].

[7] A. Falkowski, Z. Lalak and S. Pokorski, "Supersymmetrizing branes with bulk in five-dimensional supergravity," Phys. Lett. B 491, 172 (2000) [hep-th/0004093].

[8] J. Bagger and D. V. Belyaev, "Supersymmetric branes with (almost) arbitrary tensions," Phys. Rev. D 67, 025004 (2003) [arXiv:hep-th/0206024].

[9] D. V. Belyaev, "Boundary conditions in supergravity on a manifold with boundary," arXiv:hep-th/0509172.

[10] N. H. Barth, "The Fourth Order Gravitational Action For Manifolds With Boundaries," Class. Quant. Grav. 2, 497 (1985).

[11] G. W. Gibbons and S. W. Hawking, "Action Integrals And Partition Functions In Quantum Gravity," Phys. Rev. D 15, 2752 (1977).

[12] J. W. York, Jr.; "Role of conformal three-geometry in the dynamics of gravitation," Phys. Rev. Lett. 28, 1082 (1972).

[13] J. W. York, Jr.; "Boundary terms in the action principles of general relativity," Foundations of Physics, 16, 249 (1986).

[14] P. Di Vecchia, B. Durhuus, P. Olesen and J. L. Petersen, "Fermionic Strings With Boundary Terms," Nucl. Phys. B 207, 77 (1982).

[15] Y. Igarashi, "Supersymmetry And The Casimir Effect Between Plates," Phys. Rev. D 30, 1812 (1984); Y. Igarashi and T. Nonoyama, "Supergravity And Casimir Energy In A Plane Geometry," Phys. Lett. B 161, 103 (1985); Y. Igarashi and T. Nonoyama, "Supersymmetry And Reflective Boundary Conditions In Anti-De Sitter Spaces," Phys. Rev. D 34, 1928 (1986).

[16] C. Albertsson, U. Lindstrom and M. Zabzine, " $\mathrm{N}=1$ supersymmetric sigma model with boundaries. I," Commun. Math. Phys. 233, 403 (2003) [arXiv:hep-th/0111161]; C. Albertsson, U. Lindstrom and M. Zabzine, " $\mathrm{N}=1$ supersymmetric sigma model with boundaries. II," Nucl. Phys. B 678, 295 (2004) [arXiv:hep-th/0202069];

I. V. Melnikov, M. R. Plesser and S. Rinke, "Supersymmetric boundary conditions for the $\mathrm{N}=$ 2 sigma model," arXiv:hep-th/0309223; 
P. Koerber, S. Nevens and A. Sevrin, "Supersymmetric non-linear sigma-models with boundaries revisited," JHEP 0311, 066 (2003) [arXiv:hep-th/0309229].

[17] U. Lindstrom, M. Rocek and P. van Nieuwenhuizen, "Consistent boundary conditions for open strings," Nucl. Phys. B 662, 147 (2003) [arXiv:hep-th/0211266];

P. van Nieuwenhuizen and D. V. Vassilevich, "Consistent boundary conditions for supergravity," arXiv:hep-th/0507172.

[18] N. Arkani-Hamed, T. Gregoire and J. Wacker, "Higher dimensional supersymmetry in 4D superspace," JHEP 0203, 055 (2002) [arXiv:hep-th/0101233].

[19] A. Hebecker, "5D super Yang-Mills theory in 4-D superspace, superfield brane operators, and applications to orbifold GUTs," Nucl. Phys. B 632, 101 (2002) [arXiv:hep-ph/0112230].

[20] C. Csaki, C. Grojean, H. Murayama, L. Pilo and J. Terning, "Gauge theories on an interval: Unitarity without a Higgs," Phys. Rev. D 69, 055006 (2004) [arXiv:hep-ph/0305237].

[21] C. Csaki, C. Grojean, J. Hubisz, Y. Shirman and J. Terning, "Fermions on an interval: Quark and lepton masses without a Higgs," Phys. Rev. D 70, 015012 (2004) [arXiv:hep-ph/0310355].

[22] P. Horava, "Gluino condensation in strongly coupled heterotic string theory," Phys. Rev. D 54, 7561 (1996) [arXiv:hep-th/9608019].

[23] Z. Lalak and R. Matyszkiewicz, "Boundary terms in brane worlds," JHEP 0111, 027 (2001) [arXiv:hep-th/0110141].

[24] J. Wess and J. Bagger, Supersymmetry and Supergravity, 2nd Edition, Princeton University Press, 1992.

[25] I. L. Buchbinder and S. M. Kuzenko, Ideas and methods of supersymmetry and supergravity: Or a walk through superspace, Revised edition, IOP Publishing Ltd, 1998. 